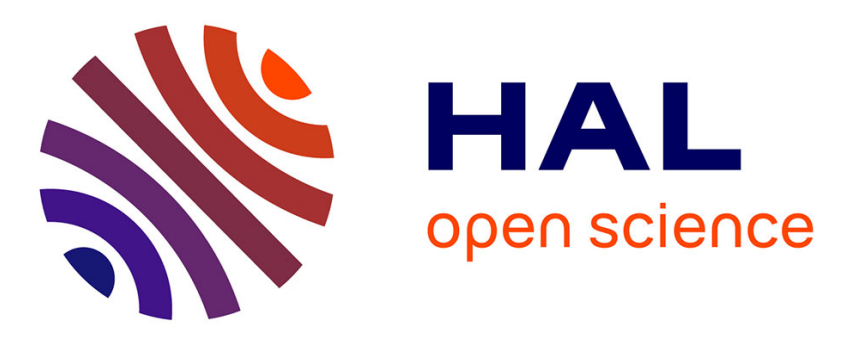

\title{
From CIE 2006 Physiological Model to Improved Age-Dependent and Average Colorimetric Observers
}

Abhijit Sarkar, Florent Autrusseau, Françoise Viénot, Patrick Le Callet, Laurent Blondé

\section{- To cite this version:}

Abhijit Sarkar, Florent Autrusseau, Françoise Viénot, Patrick Le Callet, Laurent Blondé. From CIE 2006 Physiological Model to Improved Age-Dependent and Average Colorimetric Observers. Journal of the Optical Society of America. A Optics, Image Science, and Vision, 2011, 28 (10), pp.2033-2048. 10.1364/JOSAA.28.002033 . hal-00616783

\section{HAL Id: hal-00616783 \\ https://hal.science/hal-00616783}

Submitted on 13 Mar 2013

HAL is a multi-disciplinary open access archive for the deposit and dissemination of scientific research documents, whether they are published or not. The documents may come from teaching and research institutions in France or abroad, or from public or private research centers.
L'archive ouverte pluridisciplinaire HAL, est destinée au dépôt et à la diffusion de documents scientifiques de niveau recherche, publiés ou non, émanant des établissements d'enseignement et de recherche français ou étrangers, des laboratoires publics ou privés. 


\title{
From CIE 2006 physiological model to improved age-dependent and average colorimetric observers
}

\author{
Abhijit Sarkar, ${ }^{1,2, *}$ Florent Autrusseau, ${ }^{2}$ Françoise Viénot, ${ }^{3}$ Patrick Le Callet, ${ }^{2}$ and Laurent Blondé ${ }^{1}$ \\ ${ }^{1}$ Technicolor Research \& Innovation, 1 av Belle Fontaine, CS 17616, 35576 Cesson-Sévigné Cedex, France \\ ${ }^{2}$ L'Université Nantes Angers Le Mans (LUNAM), Polytech'Nantes, Institut de Recherche en Communications \\ et Cybernétique de Nantes (IRCCyN), UMR CNRS 6597, Rue Christian Pauc, BP 50609, 44306 Nantes, France \\ ${ }^{3}$ Muséum National d'Histoire Naturelle, Centre National de la Recherche Scientifique, Ministère de la Culture et de la \\ Communication (CRCC), USR3224, 36 rue Geoffroy-Saint-Hilaire, 75005 Paris, France \\ *Corresponding author: sarkar@abhijitsarkar.com
}

Received June 10, 2011; accepted August 2, 2011; posted August 16, 2011 (Doc. ID 149015); published September 12, 2011

In the context of color perception on modern wide-gamut displays with narrowband spectral primaries, we performed a theoretical analysis on various aspects of physiological observers proposed by CIE TC 1-36 (CIEPO06). We allowed certain physiological factors to vary, which was not considered in the CIEPO06 framework. For example, we analyzed that the long-wave-sensitive (LWS) or medium-wave-sensitive (MWS) peak wavelength shift in the photopigment absorption spectra, a factor not modeled in CIEPO06, contributed more toward observer variability than some of the factors considered in the model. Further, we compared the color-matching functions derived from the CIEPO06 model and the CIE $10^{\circ}$ standard colorimetric observer to the average observer data from three distinct subgroups of Stiles-Burch observers, formed on the basis of observer ages (22-23 years, 27-29 years, and 49-50 years). The errors in predicting the $\bar{x}(\lambda)$ and $\bar{y}(\lambda)$ color-matching functions of the intragroup average observers in the long-wave range and in the medium-wave range, respectively, were generally more in the case of the CIEPO06 model compared to the $10^{\circ}$ standard colorimetric observer and manifested in both spectral and chromaticity space. In contrast, the short-wave-sensitive $\bar{z}_{10}(\lambda)$ function of the $10^{\circ}$ standard colorimetric observer performed poorly compared to the CIEPO06 model for all three subgroups. Finally, a constrained nonlinear optimization on the CIEPO06 model outputs showed that a peak wavelength shift of photopigment density alone could not improve the model prediction errors at higher wavelengths. As an alternative, two optimized weighting functions for each of the LWS and MWS cone photopigment densities led to significant improvement in the prediction of intra-age-group average data for both the 22-23 year and 49-50 year age groups. We hypothesize that the assumption in the CIEPO06 model that the peak optical density of visual pigments does not vary with age is false and is the source of these prediction errors at higher wavelengths. Correcting these errors in the model can lead to an improved age-dependent observer and can also help update the current CIE $10^{\circ}$ standard colorimetric observer. Accordingly, it would reduce the discrepancies between color matches with broadband spectral primaries and color matches with narrowband spectral primaries. (c) 2011 Optical Society of America OCIS codes: $\quad 330.0330,330.1715,330.1720,330.1730,330.5310,120.2040$.

\section{INTRODUCTION}

The most fundamental aspect of applied colorimetry is the trichromacy of our visual system, which allows us to represent any color in terms of its tristimulus values. Computing tristimulus values for any object color requires the use of the spectral reflectance of the object color, the spectral power distribution of the scene illuminant, and the spectral characteristics of a colorimetric observer. For the color imaging community, it is of interest to investigate which is a better representation of real observer data, color-matching functions (CMFs) derived from the CIE 2006 physiologically based observer model or the CIE $10^{\circ}$ standard colorimetric observer. This issue has been explored through a theoretical analysis performed in the context of display colorimetry.

\section{A. CIE $2^{\circ}$ and $10^{\circ}$ Standard Colorimetric Observers}

In 1931, the Commission Internationale de l'Éclairage (CIE) defined a standard observer for colorimetry, based on Wright's [1] and Guild's [2] $2^{\circ}$ color-matching data. However, the basic datasets were transformed to incorporate $V(\lambda)$, the luminous efficiency function of the CIE standard photometric observer [3], into the standard colorimetric observer. Incorporating both photometric and colorimetric characteristics was motivated by a need to simplify hardware computations [4], but this has been a major source of criticism of the CIE 1931 standard colorimetric observer, since the CIE standard photometric observer was based on an entirely different set of psychophysical tasks than color matching [5]. The CIE 1931 standard colorimetric observer led to spectral estimation error caused by the underestimation of luminosity at short wavelengths with the CIE standard photometric observer. Revisions of the CIE standard photometric observer $V(\lambda)$ function below $460 \mathrm{~nm}$ were proposed by Judd [6] in 1951, and further revision below $410 \mathrm{~nm}$ was proposed by Vos [7] in 1978. The former was widely accepted in the vision science community, and the latter resulted in a CIE recommendation in 1988 in the form of a supplementary observer $V_{M}(\lambda)$ for photometry [8], but the color imaging industry continued to use the original CIE 1931 standard colorimetric observer derived from $2^{\circ}$ color-matching data, applicable to small fields. 
In 1964, CIE recommended a large-field standard colorimetric observer based on the work of Stiles and Burch [9] and Speranskaya [10]. Stiles and Burch maintained high photopic luminance of the matching fields and incorporated mathematical corrections to exclude the effect of rod intrusion in long-wavelength color matches. The $\mathrm{CMF} \bar{y}(\lambda)$ represents the relative spectral luminous efficiency function of the CIE $10^{\circ}$ standard colorimetric observer, but the photometric standard still uses $\bar{y}_{2}(\lambda)$ from the CIE $2^{\circ}$ standard colorimetric observer to define luminance, even for large-field stimuli.

For many practical industrial applications, the use of the $2^{\circ}$ standard colorimetric observer is questionable, as the field of view is typically much larger than $2^{\circ}$. Indeed, many industrial engineers have chosen to use $\bar{y}_{10}(\lambda)$ in colorimetric applications. However, because of the absence of rod contribution, and more importantly, because of individual differences in the visual system, it has been observed that even the CIE $10^{\circ}$ standard colorimetric observer does not always correspond to real observer matches for large fields.

\section{B. CIE 2006 Physiologically Based Observer}

In 2006, CIE's technical committee TC 1-36 published a report [11] (described hereafter as CIEPO06, an abbreviated form of CIE 2006 physiological observer) on the choice of a set of CMFs and estimates of cone fundamentals for the colornormal observer. The CIEPO06 model is largely based on the work of Stockman and Sharpe [12]. Starting from $10^{\circ}$ CMFs of 47 Stiles-Burch observers [9] , the model defines $2^{\circ}$ and $10^{\circ}$ fundamental observers and provides a convenient framework for calculating average cone fundamentals for any field size between $1^{\circ}$ and $10^{\circ}$ and for an age between 20 and 80 .

\section{Individual Cone Fundamentals}

In its approach to construct a fundamental observer, technical committee CIE TC 1-36 has ignored individual variability $[\underline{11}, \underline{13}]$. A few studies have dealt with individual variations of $\mathrm{CMFs}$, analyzing the data collected by Stiles and Burch using $10^{\circ}$ fields [14], examining the differences between the CMFs of the CIE 1931 standard colorimetric observer, the Judd's revision of this set, and the set of $2^{\circ}$ CMFs collected by Stiles and Burch [15], comparing interindividual and intraindividual variability of experimental CMFs [16]. Wyszecki and Stiles [17] (p. 348) produced a global statistical analysis of the dispersion of the data collected by Stiles and Burch using $10^{\circ}$ fields. In the past 10 years, a few sets of matching results have been generated at low or moderate luminance levels to investigate intraobserver and interobserver variability $[18,19]$ and test additivity and transformability of color matches $[\underline{20}, \underline{21}]$. One study of nine observers' CMFs concluded that a main cause of the individual difference was the difference of individual spectral lens density [22]. Individual variations of Rayleigh matches have also been examined experimentally [23-26] or theoretically [27]. Although these studies strived to relate the variation of color matches to underlying physiological factors, they failed to model individual effects of these factors in a practical manner that could be implemented in industrial applications.

In this paper, we take advantage of the framework developed in CIEPO06 to examine through theoretical analysis the effect of age on the CMFs of individual observers and on individual color matches as viewed on displays.

\section{General Colorimetric Transforms}

Each set of CIEPO06 cone fundamentals can be converted to CMFs through a linear transformation. At the time of this work, the final $3 \times 3$ transformation matrix for such conversion was not yet made available by CIE TC 1-36. Two approaches could yield a proper linear transformation. We computed an approximate $3 \times 3$ LMS-to-XYZ transformation matrix from the available CIE $196410^{\circ} \bar{x}_{10}(\lambda), \bar{y}_{10}(\lambda), \bar{z}_{10}(\lambda)$ standard colorimetric observer functions and the average $\bar{l}_{\mathrm{SB}_{10}}^{N}(\lambda), \bar{m}_{\mathrm{SB}_{10}}^{N}(\lambda), \bar{s}_{\mathrm{SB}_{10}}^{N}(\lambda)$ cone fundamentals of 47 StilesBurch observers, each normalized to unity. The transformation matrix is

$$
\left[\begin{array}{l}
\bar{x}_{10}(\lambda) \\
\bar{y}_{10}(\lambda) \\
\bar{z}_{10}(\lambda)
\end{array}\right]=\left[\begin{array}{ccc}
1.905378 & -1.321620 & 0.419512 \\
0.698648 & 0.333043 & -0.013360 \\
-0.024300 & 0.040453 & 2.073582
\end{array}\right]\left[\begin{array}{c}
\bar{l}_{\mathrm{SB}_{10}}^{N}(\lambda) \\
\bar{m}_{\mathrm{SB}_{10}}^{N}(\lambda) \\
\bar{s}_{\mathrm{SB}_{10}}^{N}(\lambda)
\end{array}\right]
$$

The above matrix was used at all times for converting any normalized L, M, S cone fundamentals from the Stiles-Burch dataset into CIE XYZ-like CMFs similar to $10^{\circ} \bar{x}_{10}(\lambda), \bar{y}_{10}(\lambda)$, $\bar{z}_{10}(\lambda)$ functions. It is reasonably close to the matrix published earlier by other researchers [28]. Note that, in [28], a negative sign was accidentally omitted in the first row, second column of the transformation matrix [Eq. (1)].

We computed another approximate $3 \times 3$ LMS-to-XYZ transformation matrix from the $\mathrm{CIE} 10^{\circ}$ standard colorimetric observer functions and the CIEPO06 model cone fundamentals

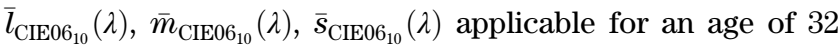
and a $10^{\circ}$ field size without any normalization of the cone fundamentals:

$$
\left[\begin{array}{c}
\bar{x}_{10}(\lambda) \\
\bar{y}_{10}(\lambda) \\
\bar{z}_{10}(\lambda)
\end{array}\right]=\left[\begin{array}{ccc}
0.006873 & -0.005386 & 0.005550 \\
0.002520 & 0.001358 & -0.000181 \\
-0.000089 & 0.000167 & 0.027432
\end{array}\right]\left[\begin{array}{c}
\bar{l}_{{\mathrm{CIE} 06_{10}}_{10}(\lambda)} \\
\bar{m}_{\mathrm{CIE} 06_{10}}(\lambda) \\
\bar{s}_{\mathrm{CIE} 06_{10}}(\lambda)
\end{array}\right] .
$$

This transformation matrix was used in the analysis of the effect of various physiological factors on CIEPO06 cone fundamentals, where normalization is not desirable. If normalized CIEPO06 cone fundamentals are used, the resulting transformation matrix is very close to that of Eq. (1).

\section{E. CIEPO06 Model}

The CIEPO06 model is a convenient and effective mathematical tool for understanding how various physiological factors affect the cone fundamentals, and thus the CMFs. A brief review of the model will be helpful in better understanding the analysis that follows.

CIEPO06 framework [11], shown in Fig. 1, involves two parameters, namely, the field size, varying between $1^{\circ}$ and $10^{\circ}$, and the observer age, varying between 20 and 80 . Three physiological factors have been incorporated in the CIEPO06 model, in the form of spectral optical density functions for: (a) lens and other ocular media absorption, (b) macular pigment absorption, and (c) visual pigments in the outer segments of photoreceptors. Out of these, the ocular media 


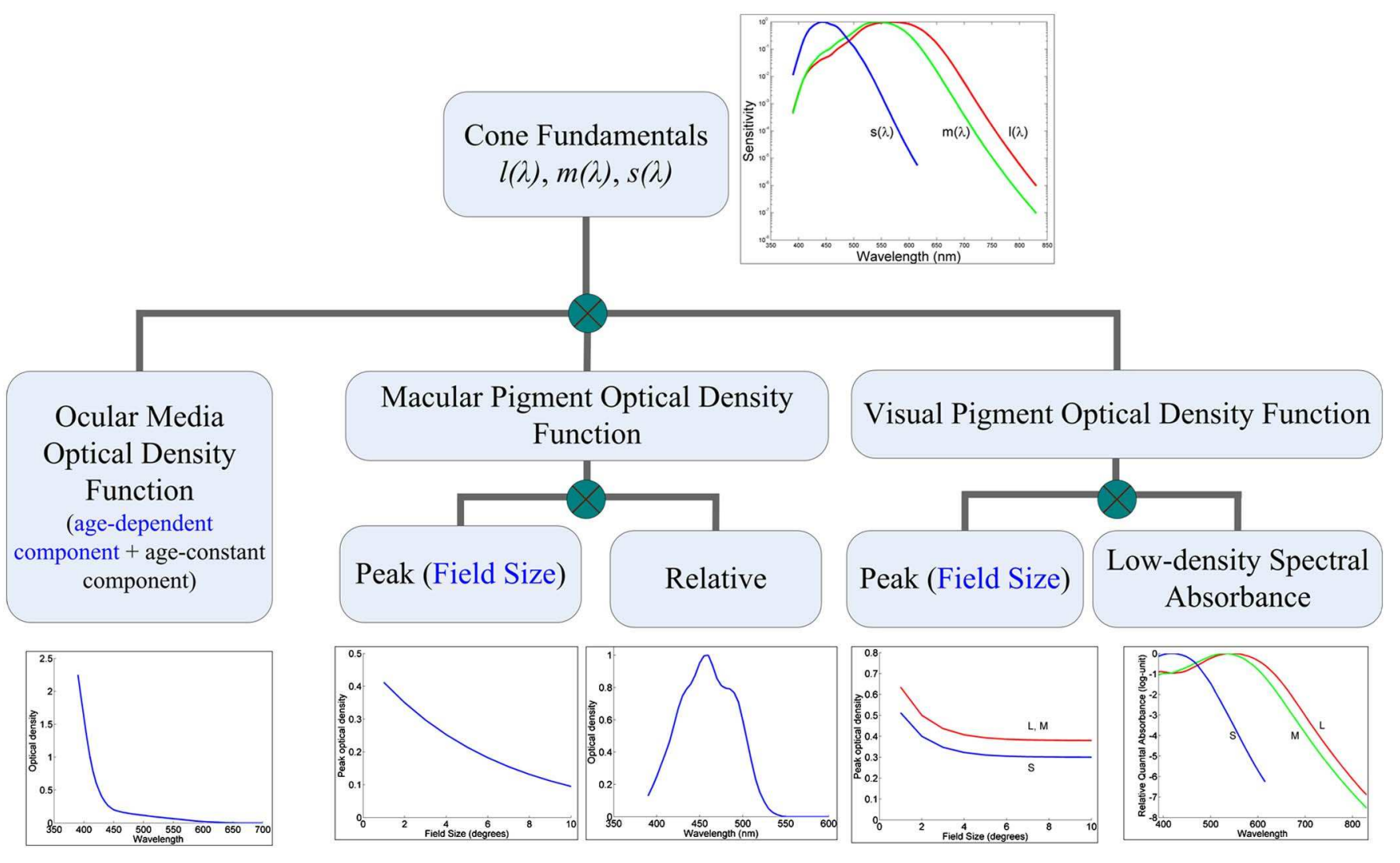

Fig. 1. (Color online) Block diagram of the CIEPO06 framework.

optical density function has an age-dependent component and an age-independent component. The macular pigment optical density function consists of a peak function and a relative function, where only the peak function varies with the field size. Similarly, the visual pigment optical density has two components, the peak as a function of the field size and the low-density spectral absorbance that is independent of any parameter.

The CIEPO06 cone fundamentals can be written in a simplified form as in Eq. (3) [11]. $A_{l}(\lambda), A_{m}(\lambda), A_{s}(\lambda)$ are the lowoptical-density spectral absorbance for long-, medium-, and short-wave-sensitive cones, respectively. $D_{\text {vis }, l}, D_{\text {vis }, m}$, and $D_{\text {vis }, s}$ are peak optical densities of the visual pigments for three cones. $D_{\text {mac }}(\lambda)$ and $D_{\text {ocul }}(\lambda)$ are the optical densities of the macular pigment and the ocular media (including the lens), respectively, with the optical density (or absorbance) being the $\log _{10}$ function of the inversed transmission of the media: $D=\log _{10}(1 / T)$ :

$$
\begin{aligned}
\bar{l}(\lambda) & =\left[1-10^{-D_{\text {vis }, l} \cdot A_{l}(\lambda)}\right] \cdot 10^{-D_{\text {mac }}(\lambda)} \cdot 10^{-D_{\text {ocul }}(\lambda),} \\
\bar{m}(\lambda) & =\left[1-10^{-D_{\text {vis }, m} \cdot A_{m}(\lambda)}\right] \cdot 10^{-D_{\text {mac }}(\lambda)} \cdot 10^{-D_{\text {ocul }}(\lambda),} \\
\bar{s}(\lambda) & =\left[1-10^{-D_{\text {vis }, s} \cdot A_{s}(\lambda)}\right] \cdot 10^{-D_{\text {mac }}(\lambda)} \cdot 10^{-D_{\text {ocul }}(\lambda) .}
\end{aligned}
$$

While these three physiological factors are important contributors to observer variability, there is another important but more complex source of variability that has not been included in the CIEPO06 model. Several studies have suggested that individual differences in color vision are partly due to the variations in the peak wavelength $\left(\lambda_{\max }\right)$ of the cone photopigment [29]. These differences can be due to individual variability, but can also be due to a variation in genetic composition or polymorphism, for example, a single amino-acid substitution (Alanine for Serine) at position 180 of the longwave-sensitive (LWS) photopigment opsin genes [30].

The rest of the paper is organized as follows. In Section $2 \underline{2}$, a theoretical analysis investigating the relative importance of various physiological factors on display color perception is presented. In Section 3 , the average Stiles-Burch observer data from three different age groups are compared with the corresponding CIEPO06 model predictions and the CIE $10^{\circ}$ standard colorimetric observer. The perceptual effect of the prediction errors in these two cases are then explored in the context of display colorimetry in Section $\underline{4}$. Next, Section $\underline{5}$ presents a constrained nonlinear optimization of the CIEPO0 $\overline{6}$ model, performed in an attempt to improve the prediction errors for various age groups. We conclude by summarizing the results obtained from these theoretical analyses in Section 6 . An exploratory analysis of the display results has been presented at a conference [31].

\section{EFFECT OF VARIOUS PHYSIOLOGICAL FACTORS ON DISPLAY COLOR PERCEPTION}

Individual variation in color perception depends on the spectral characteristics of the stimuli. As Smith and Pokorny [32] observed, "With the generally broadband spectra of reflective materials, factors such as lens transmission or macular pigment density provide correlated changes in the spectral distribution of light arriving at the retina from different samples. Thus there may be a translation of color axes but little rotation...Specification based on narrow-band trichromatic primaries may be more or less subject to individual variation, depending on the relation between the spectra of 
the biological variables and the spectra of the colorimetric primaries" (p. 27). In view of this observation, two questions arise. (i) How do various physiological factors described in Section 1 affect the color perception on a given display? (ii) How do these effects vary between a display with broadband primaries and another with narrowband primaries? The purpose of the analysis described in this section was to investigate these two issues.

\section{A. Displays Used in the Analysis}

We compared the effect of various factors in terms of color perception on two displays with different spectral characteristics. The first was a Sony BVM32 cathode ray tube (CRT) display widely used as a reference studio display (hereafter referred to as Ref-CRT). The second was a Hewlett-Packard DreamColor LP2480zx professional 30 bit wide-gamut liquid crystal display (LCD) with LED backlight (hereafter referred to as WG-LCD).

The spectral power distributions of the primaries of the two displays are shown in Fig. 2. There is a significant difference in the spectral characteristics between the two displays. WGLCD is representative of modern wide-gamut displays with peaky primaries, and Ref-CRT is representative of a typical CRT display, and of HDTV broadcasting standard references. $3 \times 3$ primary tristimulus matrices of the two displays were computed that represented the linear relationship between the XYZ tristimulus values and the RGB channel values. Note that, normally, the digital counts first need to be corrected (linearized) for the display nonlinearity (gamma correction) before computing the primary tristimulus matrix. However, since this analysis is strictly theoretical, and since gamma correction does not affect the rest of the computations, display nonlinearity has been ignored in this work. Thus, using the primary tristimulus matrix of a given display, any set of XYZ values could be converted into the corresponding set of RGB channel values and vice versa.

\section{B. Method of Analysis}

In this work, the relative importance of the four physiological factors described earlier on the cone fundamentals were

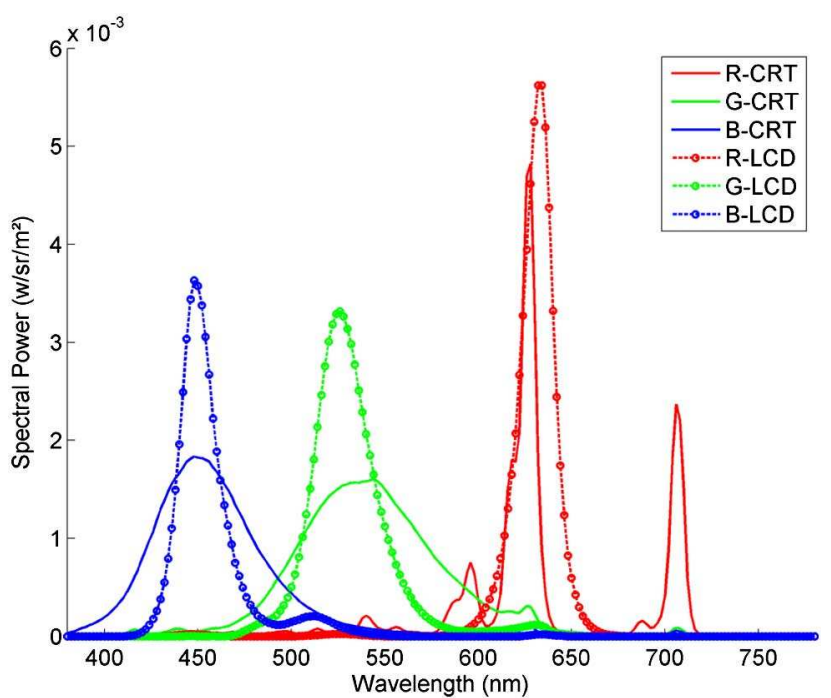

Fig. 2. (Color online) Spectral power distributions of the two displays used in the analysis. explored within the framework of CIEPO06. Cone fundamentals for $10^{\circ}$ field size and an observer age of 32 were computed by independently modifying the contribution of individual factors. In the first analysis published previously [31], we completely removed the contribution of various factors one at a time, by setting the optical density term to zero in case of ocular media and macular pigment absorption, by setting the peak optical density to unity in the case of low-density absorption spectra (signifying very high absorption taking place in the outer segments of visual pigments), and by shifting the peak wavelength of the LWS photopigment optical density by $250 \mathrm{~cm}^{-1}$ toward shorter wavelengths in case of peak wavelength shift. In the second analysis presented in this paper, we independently modified the contributions of various physiological factors as follows:

i. mean optical density of ocular media varied by $\pm 25 \%$,

ii. peak optical density of macular pigment varied by $\pm 25 \%$,

iii. peak optical density term for low-density photopigment relative absorption spectra varied by $\pm 25 \%(0.38$ is nominal), and

iv. peak wavelength shift of the cone photopigment optical density in the outer segment of the photoreceptor: (a) LWS peak shift by $-4 \mathrm{~nm}$ (toward a shorter wavelength) and (b) medium-wave-sensitive (MWS) peak shift by $+4 \mathrm{~nm}$ (toward a longer wavelength).

Such modifications of optical densities by the same percentage allow us to compare the effect of various factors. For cases (i) and (iv), the modifications are the same as those reported by Smith and Pokorny [32]. A high optical density in case (iii) signifies higher photoreceptor self-screening, resulting in the broadening of the photopigment relative absorption spectra [33] (pp. 65-66), while case (iv) signifies LWS and MWS polymorphism described earlier. For case (iv), the peak wavelength $\lambda$ was first shifted in the wavenumber scale $\left(\nu=10^{7} / \lambda\right.$, where $\nu$ is in $\mathrm{cm}^{-1}$ and $\lambda$ is in nanometers), the cone absorptance spectra were resampled, modified cone fundamentals were computed and converted from the quanta to energy units, and, finally, were renormalized. Note that case (iv) considers LWS and MWS peak wavelength shifts independently.

For each planned variation of these four factors, a set of modified CIEPO06 cone fundamentals was computed, and were compared to corresponding CIEPO06 cone fundamentals under normal conditions. The difference between the two sets of functions indicates the contribution of a given physiological factor. The difference was computed in terms of Euclidean distance in the cone fundamental space. Note also that we use CIEPO06 $10^{\circ}$ cone fundamentals, unlike Smith and Pokorny $2^{\circ}$ cone fundamentals as in [32].

In order to simulate the effect of various physiological factors when viewing color stimuli on different displays, chromaticities of these stimuli for a given display and a given set of modified CIEPO06 cone fundamentals must be computed. In this analysis, seven test stimuli were selected from various parts of the common gamut of the CRT and the LCD. These stimuli were chosen such that they covered the whole common display gamut in the CIE $1976\left(u^{\prime}, v^{\prime}\right)$ coordinate system (Fig. 3). The chromaticity of the seventh stimulus was close to that of display white. These coordinates were converted to the 


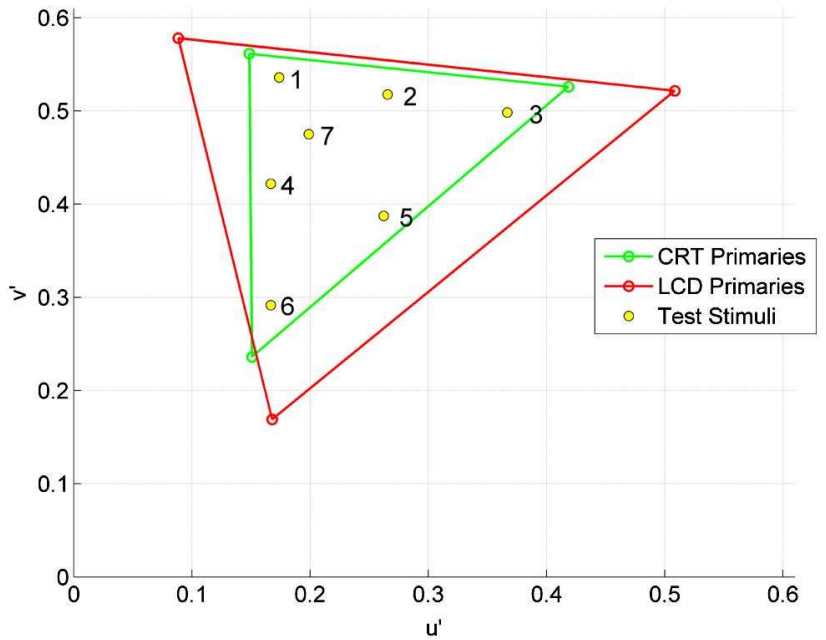

Fig. 3. (Color online) Seven test stimuli in $\left(u^{\prime}, v^{\prime}\right)$ chromaticity diagram.

XYZ colorimetric system through a straightforward transformation, as shown in Eq. (ㅁ). The chromaticity coordinates are listed in Table $\underline{1}$ :

$$
\begin{aligned}
x & =\frac{9 u^{\prime}}{6 u^{\prime}-16 v^{\prime}+12}, \quad y=\frac{4 v^{\prime}}{6 u^{\prime}-16 v^{\prime}+12}, \\
X & =\frac{x}{y} Y, \quad Z=\frac{z}{y} Y .
\end{aligned}
$$

Smith and Pokorny [32] investigated the effects of different physiological factors on two sets of chromaticities at a nominal luminance of $8 \mathrm{~cd} / \mathrm{m}^{2}$, varying along the horizontal and vertical lines in the cone-troland chromaticity diagram. This luminance level is rather low for most industrial applications, thus we used a constant luminance of $25 \mathrm{~cd} / \mathrm{m}^{2}$ for seven distinct chromaticities described in Subsection 2.C. For our application context (content production for the film industry), we considered this an appropriate luminance level for the displays.

From tristimulus values $\left(X_{10}, Y_{10}, Z_{10}\right)$ of the test stimuli, the RGB channel values $(R, G, B)$ required to produce these colors on the two displays were computed using the display primary tristimulus matrices, as shown in Eq. (5). The primary tristimulus matrix for a display is formed by the tristimulus values of peak primaries:

$$
\left[\begin{array}{c}
R \\
G \\
B
\end{array}\right]=\left[\begin{array}{lll}
X_{r, \max } & X_{g, \max } & X_{b, \max } \\
Y_{r, \max } & Y_{g, \max } & Y_{b, \max } \\
Z_{r, \max } & Z_{g, \max } & Z_{b, \max }
\end{array}\right]^{-1} *\left[\begin{array}{c}
X_{10} \\
Y_{10} \\
Z_{10}
\end{array}\right] .
$$

The product of the RGB values for each channel and the spectral data of the corresponding display primaries $\left[P_{\text {pri- } R}(\lambda)\right.$, $\left.P_{\text {pri- } G}(\lambda), P_{\text {pri- } B}(\lambda)\right]$, when added for all three channels, gave the spectral power distribution of the test stimuli for a given display, as per Eq. (6). These spectral data were used to compute tristimulus values in the subsequent step, described next. In computing the spectral power distribution of the test stimuli, we assume the displays have perfect additivity and proportionality, and also stable primaries:

$$
P_{\text {stim }}(\lambda)=\left[\begin{array}{lll}
R & G & B
\end{array}\right] *\left[\begin{array}{c}
P_{\mathrm{pri}-R}(\lambda) \\
P_{\mathrm{pri}-G}(\lambda) \\
P_{\mathrm{pri}-B}(\lambda)
\end{array}\right] .
$$

\section{Derivation of Cone-Troland Coordinates from a Given Set of Display Channel Values}

The derivation of cone-troland coordinates from SmithPokorny $2^{\circ}$ cone fundamentals has been described in detail elsewhere [34-36]. The method used in this study for deriving the cone-troland coordinates corresponding to a given set of display channel values and the CIEPO06 $10^{\circ}$ cone fundamentals is described now.

MacLeod and Boynton [36] proposed a chromaticity diagram $\left(l_{\mathrm{MB}}, s_{\mathrm{MB}}\right)$ [see Eq. (7)], where the projective plane is an equiluminant chromaticity plane. A basic assumption in forming the MacLeod-Boynton chromaticity diagram is that short-wavelength-sensitive (SWS) cone fundamental $\bar{s}(\lambda)$ does not contribute to luminance. In this diagram, the abscissa $l_{\mathrm{MB}}=L /(L+M)$ represents the equal and opposite change in LWS and MWS cone excitations, i.e., an increase in the LWS luminance is counterbalanced by an equal decrease in MWS luminance, but the sum is unity. The ordinate $s_{\mathrm{MB}}=$ $S /(L+M)$ denotes the level of SWS cone excitation:

$$
l_{\mathrm{MB}}=\frac{L}{L+M}, \quad s_{\mathrm{MB}}=\frac{S}{L+M} .
$$

In order to scale the ordinate axis, the concept of cone trolands has been introduced. Since the troland is a unit used to express a quantity proportional to retinal illuminance, the amount of $L$-cone trolands and $M$-cone trolands indicates the respective contribution of LWS and MWS cone excitations to the retinal illuminance. Since SWS cones do not contribute

Table 1. CIE $1964 x y$ and CIE $1976\left(u^{\prime}, v^{\prime}\right)$ Chromaticity Coordinates for Seven Test Stimuli and the Display Whites

\begin{tabular}{cccccc}
\hline Stimulus & $x_{10}$ & $y_{10}$ & $Y\left(\mathrm{~cd} / \mathrm{m}^{2}\right)$ & $u_{10}^{\prime}$ & 0.1737 \\
\hline TS-1 & 0.35 & 0.48 & 25 & 0.2655 & 0.536 \\
TS-2 & 0.45 & 0.39 & 25 & 0.3668 \\
TS-3 & 0.53 & 0.32 & 25 & 0.1667 & 0.5177 \\
TS-4 & 0.24 & 0.27 & 25 & 0.2623 \\
TS-5 & 0.32 & 0.21 & 25 & 0.1667 \\
TS-6 & 0.18 & 0.14 & 25 & 0.1988 & 0.4219 \\
TS-7 & 0.32 & 0.34 & 25 & 0.1966 & 0.3873 \\
Full white CRT & 0.3093 & 0.3260 & 97.36 & 0.1957 \\
Full white LCD & 0.3070 & 0.3240 & 97.01 & 0.4662 \\
\hline
\end{tabular}


to luminance, $S$-cone troland must be appropriately defined. In the representation proposed by Boynton and Kambe [37], one troland of the equal-energy spectrum amounts to one $S$ cone troland.

In that case of CIEPO06 cone fundamentals that are the same as Stockman-Sharpe $10^{\circ}$ cone fundamentals, each scaled to unity peak, the luminous efficiency function is given by Eq. (ㅇ) [38]. However, as our analysis involves comparing normal and modified cone fundamentals, any normalization must be avoided since it can unduly shift the peak wavelength of modified cone fundamentals, making it difficult to infer whether such shift is due to a physiological factor or because of normalization.

When cone fundamentals are not normalized to unity peak, the luminous efficiency function can be obtained by adding LWS and MWS cone fundamentals in a 1.98:1 ratio [same ratio as in Eq. (8)]; thus LWS cone fundamentals were scaled by 1.98 to begin with [Eq. (9)]. No scaling was used for SWS cone fundamentals. Next, the product of scaled cone fundamentals and the test stimulus spectral power distribution $\left[P_{\text {stim }}(\lambda)\right]$ obtained from Eq. (6) were computed for each wavelength and summed over the whole wavelength range, resulting in LMS tristimulus values in the cone fundamental space [Eq. (10)]. The resulting tristimulus values were specific to a given display and a given set of modified cone fundamentals, computed from various normal and modified CIEPO06 $10^{\circ}$ cone fundamentals. Macleod-Boynton chromaticity coordinates $\left(l_{\mathrm{MB}}\right.$, $s_{\mathrm{MB}}$ ) were then obtained from LMS tristimulus values as described before [Eq. (7)]:

$$
\begin{gathered}
V_{\mathrm{SS}, 10}(\lambda)=0.692839 \bar{l}(\lambda)+0.349676 \bar{m}(\lambda), \\
l_{\mathrm{SC}}(\lambda)=\bar{l}(\lambda) * 1.98, \\
{\left[\begin{array}{c}
L \\
M \\
S
\end{array}\right]=\left[\begin{array}{c}
l_{\mathrm{SC}}(\lambda) \\
m(\lambda) \\
s(\lambda)
\end{array}\right] * P_{\text {stim }}(\lambda) .}
\end{gathered}
$$

Again, to comply with the definition of $S$-cone trolands, Macleod-Boynton $s$ coordinates $\left(s_{\mathrm{MB}}\right)$ were scaled such that $s$ coordinate of equal-energy white would be equal to unity. In the case of CIEPO06 $10^{\circ}$ cone fundamentals, the computed scale factor was 21.7209 .

The luminance values $\left[Y_{\text {stim }}\right]$ of the test stimuli were obtained by vectorially adding the peak primary luminance values $\left[Y_{R \max }, Y_{G \max }, Y_{B \max }\right]$ scaled by the respective channel values, as shown in Eq. (11):

$$
Y_{\text {stim }}=\left[\begin{array}{lll}
R & G & B
\end{array}\right] *\left[\begin{array}{c}
Y_{R \max } \\
Y_{G \max } \\
Y_{B \max }
\end{array}\right] .
$$

Using the above method, relative cone trolands were computed for the seven test stimuli and are plotted in Fig. 4.

Using an observer model different from the $10^{\circ}$ standard colorimetric observer is likely to distort the uniformity of $u^{\prime} v^{\prime} Y$ color space, the extent of which depends on the specific observer model used. However, in our analysis we hypothesize that, in a small region of three-dimensional space around

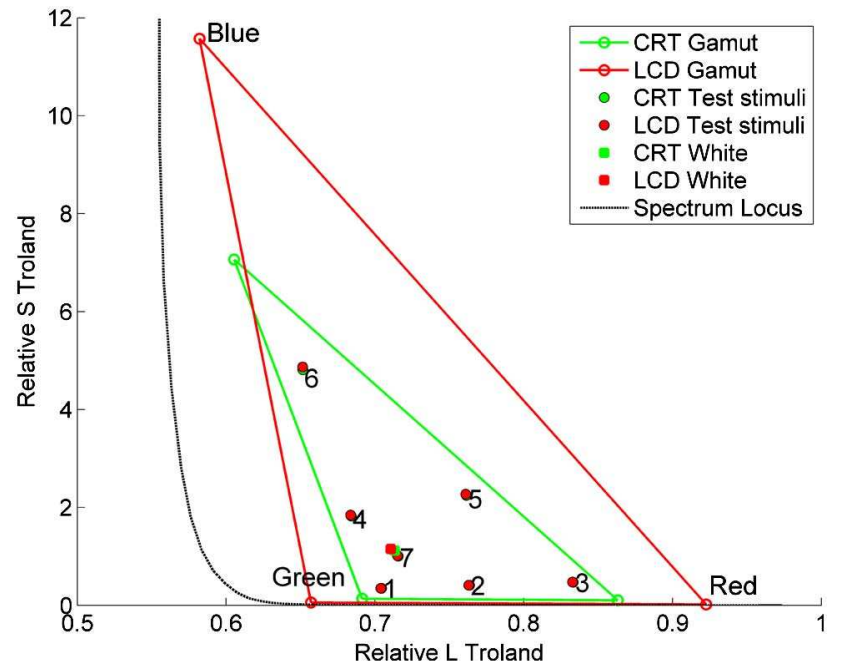

Fig. 4. (Color online) Seven test stimuli in Boynton-Kambe relative cone-troland coordinates based on CIEPO06 $10^{\circ}$ cone fundamentals.

a given color, the Euclidean distances for various observer CMFs can be compared. Because of this issue, use of a more complex color space like CIELAB and color difference equations was avoided as they could possibly amplify uniformity distortions. $u^{\prime} v^{\prime} Y$ was chosen over $x y Y$ because of better visual uniformity.

\section{Results}

In Fig. $\underline{5}$, the $\left(u^{\prime}, v^{\prime}\right)$ chromaticity shifts of the seven test stimuli are shown, depicting the effects of modified cone fundamentals on color perception on the CRT (light green symbols) and the LCD (dark red symbols). The squares represent a $25 \%$ increase in the optical density of the ocular media [Fig. 5(a)], of the macular pigment [Fig. 5(b)], and of the cone photopigment [Fig. 5(c)], and in Fig. $5(\mathrm{~d})$, a shift of the peak LWS cone wavelength by $4 \mathrm{~nm}$ toward shorter wavelengths (see Subsection 3.B). The triangles represent a $25 \%$ decrease in the optical density of the ocular media [Fig. 5(a)], of the macular pigment [Fig. 5(b)] and of the cone photopigment [Fig. 5(c)], and in Fig. 5(d), a shift of the peak MWS cone wavelength by $4 \mathrm{~nm}$ toward longer wavelengths. Figure 6 shows the same chromaticity shifts in the cone-troland chromaticity diagram. Table 2 represents the root mean square (RMS) of the $\left(u^{\prime}\right.$, $v^{\prime}$ ) coordinate shifts of seven displayed stimuli due to each of the four factors (scaled by 1000). Mean and maximum RMS differences are shown for both displays.

All four factors do not affect the target specification to the same extent. Out of all four factors, photopigment peak optical density affects the observer color perception the most, as evident from Table 2 . In the case of ocular media and macular pigment absorption, the change in color perception occurs along the same direction: toward yellow-green when the optical density is increased and toward blue when it is decreased [Figs. 5(a) and 5(b)]. This is true even for the test stimulus close to the display white. These directions of change are in line with Wyszecki and Stiles' results [17] (p. 352). However, the effect of macular pigment absorption is significantly less than ocular media absorption; in fact, it is the least significant physiological factor when compared to the others, as per Table 2. The change due to macular pigment absorption is marginally larger for LCD as compared to the CRT, and is the opposite in the case of ocular media absorption. That ocular 

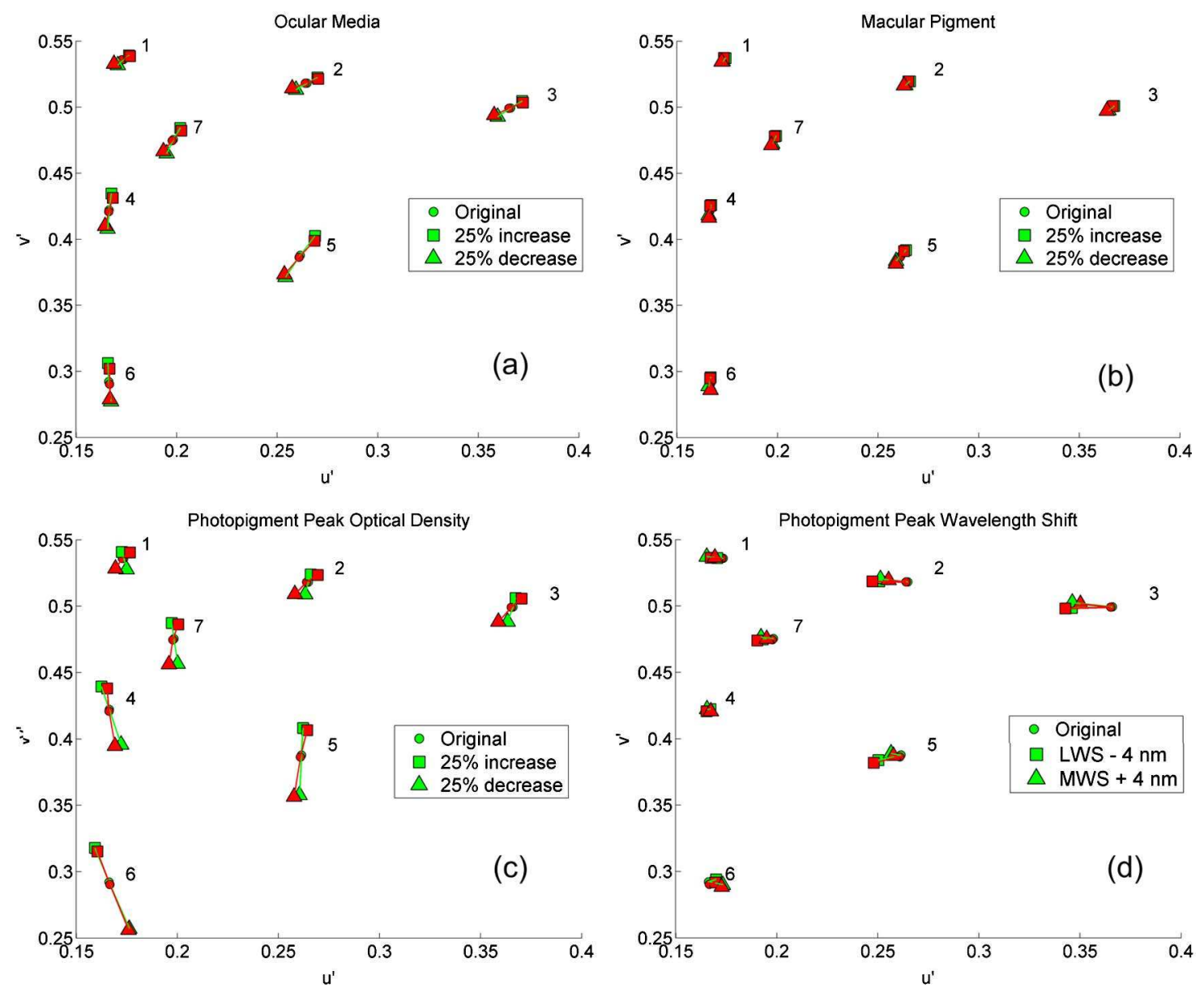

Fig. 5. (Color online) Simulated chromaticity shift for seven test stimuli due to modified cone fundamentals in $\left(u^{\prime}, v^{\prime}\right)$ chromaticity diagram. The circles show the original chromaticities of the stimuli. Increase (squares) and decrease (triangles) of the peak optical density by $25 \%$ are shown (a) for ocular media, (b) for macular pigment, and (c) for photopigment peak optical density.(d) Peak wavelength shift of LWS cone photopigment by $4 \mathrm{~nm}$ toward shorter wavelengths (squares) and of MWS cone photopigment by $4 \mathrm{~nm}$ toward longer wavelengths (triangles). Light green symbols correspond to the CRT and dark red symbols to LCD.

media optical density plays a dominant role in observer variability, even within the same age group, has been reported by several vision researchers. Pokorny et al. [39] observed that "studies which include a large number of observers of similar age indicate that there is considerable variability in estimated lens density at any given age. For example, van Norren and Vos noted that the difference between the five highest and five lowest of Crawford's observers was greater than one log unit at $400 \mathrm{~nm}$. This variation may be even more pronounced in an older group of subjects" (pp. 1438-1439). Note that, in terms of cone excitation, the largest change due to modification of ocular media and macular pigment absorption occurs for the blue color (test stimulus 6).

Finally, in case of CRT, the effect of photopigment peak wavelength shift is as large as that of ocular media absorption, particularly the LWS cone shift. In the case of LCD, the LWS cone peak wavelength shift is by far the second most important factor in influencing display color perception, after photopigment peak optical density.

\section{E. Analysis of Results}

Interestingly, the direction of change due to the modification of the photopigment peak optical density is different for the CRT and the LCD, both in terms of $\left(u^{\prime}, v^{\prime}\right)$ chromaticity coordinates [Fig. 5(c)] and relative cone trolands [Fig. 6(c)]. This difference is more apparent in the green-red region of color space and reduces as we go toward blue. We can assume that relative position of display primaries with respect to the cone fundamentals has an influence on such difference in directional effects between the two displays. However, other physiological factors do not show such trend. Another observation is that for the reddish-yellow (test stimulus \#2), red (test stimulus \#3), and magenta (test stimulus \#5), the directions of change due to LWS and MWS peak wavelength shifts [Figs. 5(d) and 6(d)] are the same. An explanation of this observation is that the LWS and MWS peaks move toward each other. For other stimuli, the effect of peak wavelength shifts is not significant.

Since the photopigment peak optical density has the strongest influence in display color perception compared to other factors, and since the largest chromaticity shift due to this factor occurs in blue, we can assume that individual variations in the color vision of a large population of real observers will have a significant impact on the perception of blue.

This analysis also shows that the photopigment peak wavelength shift is an important physiological factor affecting display color perception, particularly in the case of modern displays with narrowband primaries (Table 2). The difficulty in modeling this factor imposes serious limitation on the agedependent observers of CIEPO06. Observer variability within 

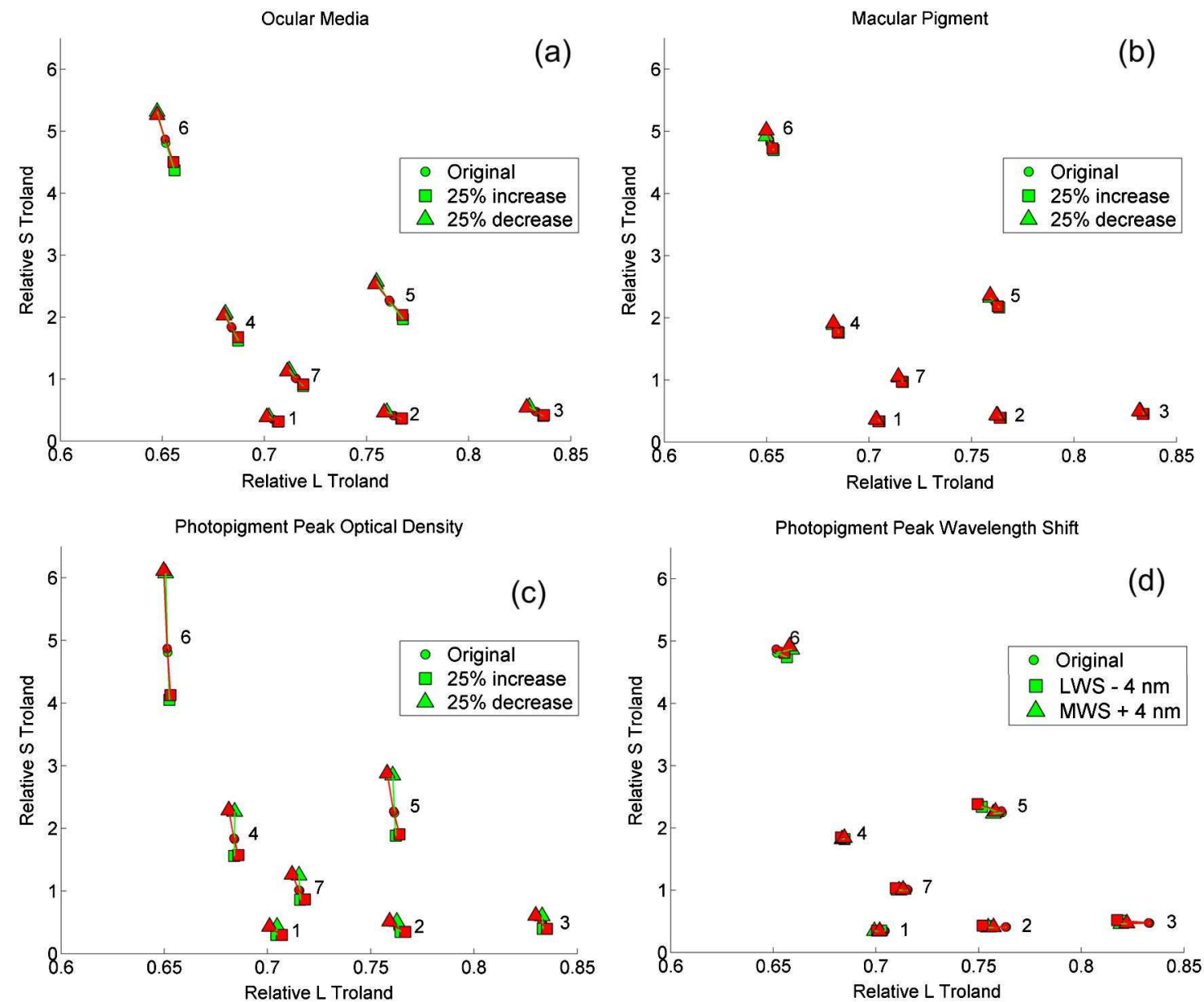

Fig. 6. (Color online) Simulated chromaticity shift for seven test stimuli due to modified cone fundamentals in relative cone-troland space. The circles show the original chromaticities of the stimuli. Increase (squares) and decrease (triangles) of the peak optical density by $25 \%$ are shown (a) for ocular media, (b) for macular pigment, and (c) for photopigment peak optical density. (d) Peak wavelength shift of LWS cone photopigment by $4 \mathrm{~nm}$ toward shorter wavelengths (squares) and of MWS cone photopigment by $4 \mathrm{~nm}$ toward longer wavelengths (triangles). Light green symbols correspond to the CRT and dark red symbols to LCD.

a given age group due to such factors cannot be predicted, even though this variability can be more significant than the effects of some of the factors already included in the model.

This analysis has some inevitable constraints. It is difficult to predict the extent to which various physiological factors affect the color perception of an individual observer. It is also difficult to ascertain what amount of peak wavelength shift should physiologically correspond to a $25 \%$ change in peak optical densities. We chose a peak wavelength shift of $4 \mathrm{~nm}$ since this is the largest shift observed due to the serinealanine amino acid substitution at position 180 of the photopigment opsin genes, a common form of polymorphism [30]. We hypothesize that the conditions analyzed here all represent extreme changes in four physiological factors, and thus are reasonable to compare. In spite of the above constraint, this analysis highlights the relative importance of various factors in affecting color perception on displays.

\section{INTRA-AGE-GROUP AVERAGE OBSERVER PREDICTION WITH CIEPOO6 MODEL AND THE CIE $10^{\circ}$ STANDARD COLORIMETRIC OBSERVER}

Based on the foregoing discussion, a question arises whether it could be worthwhile to explore if the observed intersubject differences in color matches could be predicted by adjustment of more of the CIEPO06 parameters. In this study, experimental data from the 1959 Stiles-Burch study [9] involving 47 observers were re-examined, since this is the most comprehensive visual dataset for color vision available to date.

\section{A. CIEPO06 Age Parameters for Real Observers}

The age parameter was introduced in the CIEPO06 model to take into account the difference in absorption in the ocular media, in particular the lens, between the aged and the young observers. At that time, the age dependencies of the absorption by the macular pigment as well as the densities of the visual pigments were considered of minor influence. The two-component age function of the CIEPO06 model originated from several experimental bases that were thought to be representative of large groups of observer [39]. Thus, the CIEPO06 age parameter does not necessarily correspond to the age of the real Stiles-Burch observers. In other words, predicted model functions that best match the real observer data may not always be obtained using real observer ages. This may happen because of random observer variability, and/or because of the exclusion of one or more physiological factors from the CIEPO06 model. These factors could be age independent, like the peak wavelength shift of the LWS or MWS cone 
Table 2. $\left(u^{\prime}, v^{\prime}\right)$ RMS Distance $(\times 1000)$ from Average Cone Fundamental

\begin{tabular}{|c|c|c|c|c|c|}
\hline Source of Variability & & \multicolumn{2}{|c|}{ RMS $(\times 1000)[C R T]$} & \multicolumn{2}{|c|}{ RMS $(\times 1000)[\mathrm{LCD}]$} \\
\hline Ocular media peak optical density & $0.25 \%$ & 10.25 & 16.45 & 9.40 & 14.53 \\
\hline \multirow[t]{2}{*}{ Macular pigment peak optical density } & $0.25 \%$ & 2.93 & 4.69 & 3.25 & 5.04 \\
\hline & $-0.25 \%$ & 2.96 & 4.72 & 3.29 & 5.08 \\
\hline Photopigment peak optical density & $0.25 \%$ & 13.51 & 26.59 & 13.85 & 25.60 \\
\hline Photopigment peak wavelength shift & $M+4 \mathrm{~nm}$ & 8.72 & 20.01 & 5.84 & 15.09 \\
\hline
\end{tabular}

photopigment as discussed in Subsection 2.B, or these could be age-dependent physiological factors not considered in CIEPO06. CIE committee TC 1-36 also recognized this restriction by pointing out that the CIEPO06 fundamental observer was a theoretical construct [11]. In this analysis, we determined the CIEPO06 age parameters that resulted in the best predictions of each individual Stiles-Burch observer cone fundamental data. For each individual Stiles-Burch observer, three CIEPO06 age parameters were derived so as to fit as closely as possible the three cone fundamentals, respectively. Two different methods were used. In the first method, we computed the correlation coefficients between the normalized cone fundamentals for each Stiles-Burch observer, using Eq. (1) as explained in Subsection 1.D, and those corresponding to all possible CIEPO06 age parameter values between 20 and 80 (a total of 61). The corresponding CIEPO06 age was the one yielding the highest correlation coefficient for a given cone fundamental. This process was repeated for all three cone fundamentals and for all 47 Stiles-Burch observers. In the second method, the corresponding CIEPO06 age for each Stiles-Burch observer was predicted by minimizing the RMS errors between the normalized cone fundamentals for each Stiles-Burch observer, and those corresponding to all possible CIEPO06 age parameter values between 20 and 80 .

\section{B. Comparison of CIEPO06 Predicted and Real Ages of Stiles-Burch Observers}

In Fig. 7, the CIEPO06 predicted ages obtained using the correlation coefficient (CORR) method have been plotted against

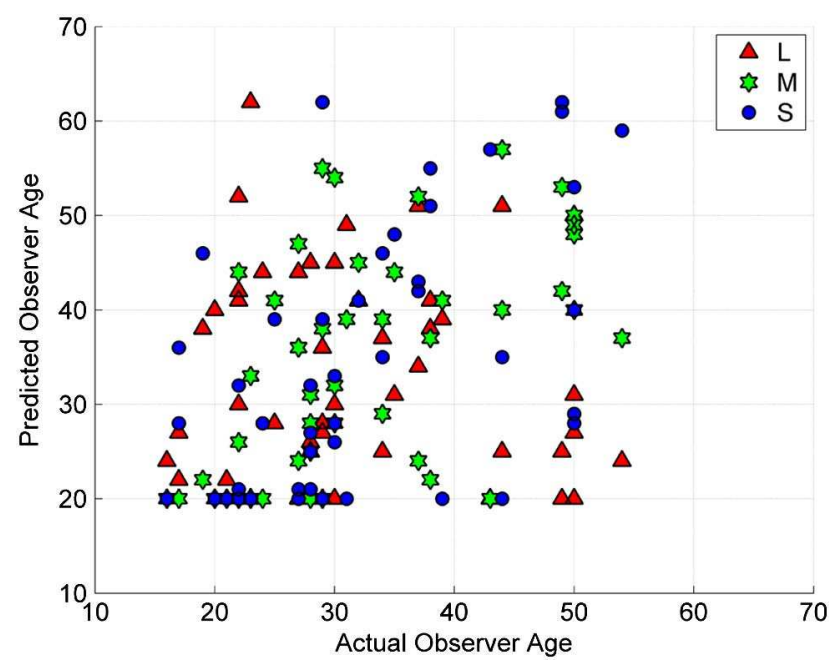

Fig. 7. (Color online) Age correspondence between CIEPO06 model's best prediction and 47 Stiles-Burch observers. the actual ages of 47 Stiles-Burch observers. The second method (RMS error, RMSE) produced very similar results. No direct correspondence was found between the real and predicted ages.

The gain offered by the adjusted CIEPO06 age over the real age could be validated by examining the prediction of matches of equal-energy white. Figure $\underline{8}$ shows $(x, y)$ chromaticity of equal-energy white computed with CMFs derived from CIEPO06 cone fundamentals for each Stiles-Burch observer. CIEPO06 cone fundamentals were obtained by using corresponding ages from both methods (CORR and RMSE) as well as by using actual observer ages. Matches obtained with real observer cone fundamentals are also plotted. While CIEPO06 with age correspondence (with either method) yields greater observer variability than CIEPO06 with actual observer ages, it fails to explain all the variability in the real observer data, particularly along the ordinate.

The mean standard deviations of the CIEPO06 cone fundamentals from the 47 Stiles-Burch observer data averaged over all observers are plotted in Fig. 9. The LWS, MWS, and SWS cone fundamentals obtained by using corresponding ages from the two methods (CORR and RMSE) and by using actual observer ages are shown. Mean (central mark), as well as the 25th and 75th percentiles (dotted bars) of standard deviations are higher when real observer ages are used in the model. The error is higher for LWS and MWS cone fundamentals than for the SWS cone fundamental. This further shows that, by

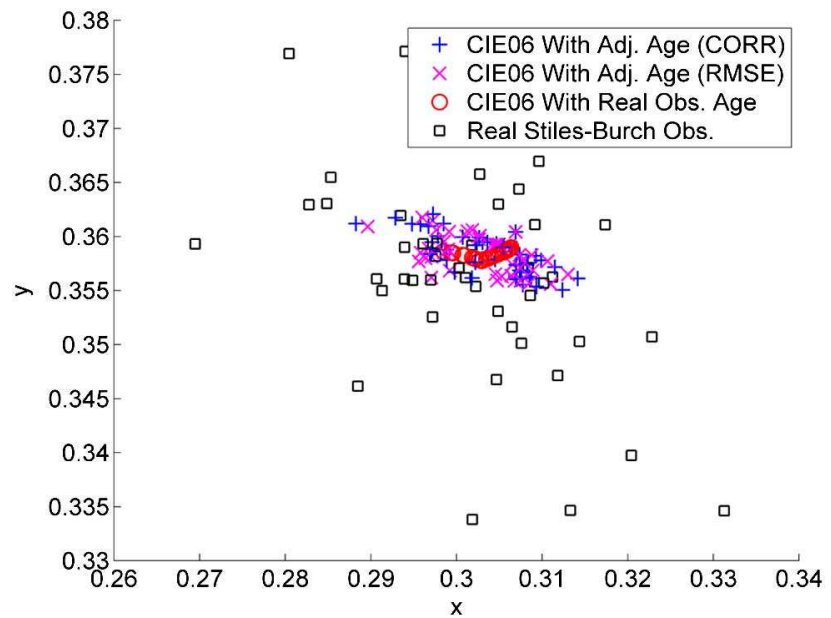

Fig. 8. (Color online) Chromaticities of matches of equal-energy white, computed using cone fundamentals from the 47 Stiles-Burch observer data and CIEPO06 predictions, with two adjustment methods for age (CORR and RMSE) as well as with actual observer age. 


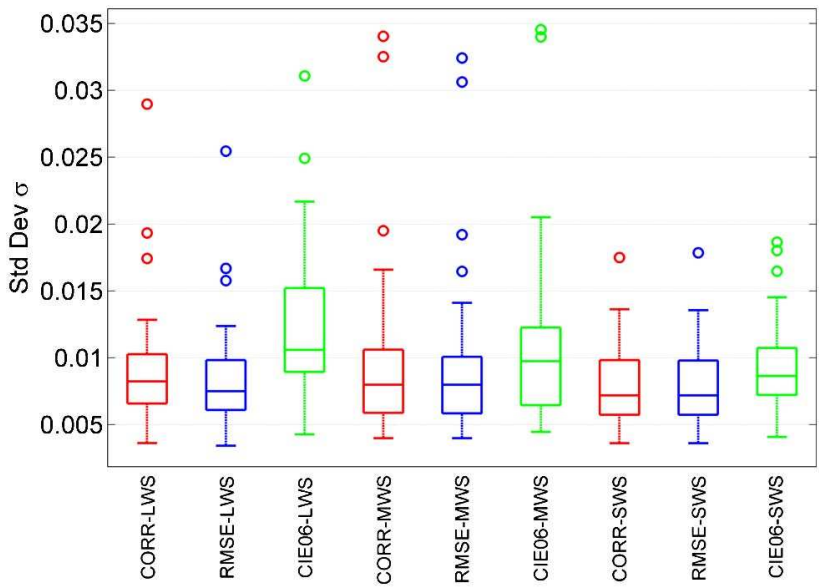

Fig. 9. (Color online) Mean standard deviation of CIEPO06 cone fundamentals from the 47 Stiles-Burch observer data, with two adjustment methods for age (CORR and RMSE) as well as with actual observer age. On each box, the central mark is the median, the edges of the box are the 25th and 75th percentiles, and the whiskers extend to the most extreme data points that are not considered outliers, while outliers are plotted individually as small circles.

adjusting the age parameter, the CIEPO06 prediction of real Stiles-Burch observer data is improved.

\section{Grouping Stiles-Burch Observers with Respect to Age} To conform to the age-dependent observer model of CIEPO06, three dominant age groups among the Stiles-Burch observers were identified. The groups were formed in such a way that the age difference between observers within any group was not more than 2 years. This constraint allowed grouping of only 22 out of 47 observers. Six observers with ages between 22 and 23 formed Group 1, ten observers with ages between 27 and 29 formed Group 2, and another six observers with ages between 49 and 50 were placed in Group 3. In the rest of the analysis, these three observer groups are used. For each group, CIEPO06 age correspondence for the average data was established using the correlation coefficient method for the average Stiles-Burch cone fundamentals for the group and CIEPO06 cone fundamentals for all possible ages. In the following sections, we obtained two sets of CIEPO06 CMFs for each observer group, CIEPO06 CMFs obtained by using adjusted age parameter values given by the correlation coefficient method and CIEPO06 CMFs obtained by using actual average observer ages.

\section{Comparing CIEPO06 Model Prediction and $10^{\circ}$ Standard Colorimetric Observer with Intragroup Average}

Once three groups of observers were identified, the variability of CMFs was examined within each group. The examination put more emphasis on the regions of the spectrum where $\bar{x}_{10}(\lambda), \bar{y}_{10}(\lambda), \bar{z}_{10}(\lambda)$ peak. In Fig. 10 , intragroup minimum, maximum, and average CMF values are shown along with the $10^{\circ}$ standard colorimetric observer CMFs, the CIEPO06 model predictions, and with age correspondence and with real ages. Table 3 lists the results of a statistical comparison of the Stiles-Burch observer CMFs, $10^{\circ}$ standard colorimetric observer, and CIEPO06 model predictions with age correspondence and with real ages. Values corresponding to $\bar{x}_{10}(\lambda), \bar{y}_{10}(\lambda)$, $\bar{z}_{10}(\lambda)$ functions, in the corresponding long-, medium-, and short-wavelength ranges for each group, are shown. The third column in Table 3 shows the intragroup standard deviation of the Stiles-Burch data (note that standard deviation has the same units as the data), signifying intragroup observer variability. The following three columns list absolute difference of various functions from the intragroup mean, averaged over all wavelengths. The three functions considered here are (i) the $10^{\circ}$ standard colorimetric observer, (ii) CIEPO06 with real observer ages as input, and (iii) adjusted CIEPO06 ages with age correspondence as input. The absolute differences of the functions were multiplied by three weighting functions (for LWS, MWS, and SWS, respectively) before averaging over all wavelengths. The weighting functions were computed by dividing the three intragroup average Stiles-Burch observer CMFs by their respective sum over all wavelengths. The role of the weighting functions was to assign more weights to the values around the peak than those in the lower end of the ordinate, while ensuring the weights were proportional to original observer data. Note that, since the $\bar{x}_{10}(\lambda), \bar{y}_{10}(\lambda), \bar{z}_{10}(\lambda)$ CMFs do not have the same ordinate scale, the rows should not be compared as such.

As shown in Fig. 10 and Table $\underline{3}$, in the case of $x$ CMFs for Groups 1 and 3, both the original CIEPO06 model predictions with real ages and the $10^{\circ}$ standard colorimetric observer deviate from the intragroup average. The CIEPO06 model with real observer ages generally performs similar to or worse than the $10^{\circ}$ standard colorimetric observer $\bar{x}_{10}(\lambda)$ and $\bar{y}_{10}(\lambda)$ CMFs. For Groups 1 and 3, the age correspondence method mostly improves CIEPO06 predictions, and is mostly better than the standard colorimetric observer. For Group 2, the prediction error is relatively low, even without age correspondence, indicating that the CIEPO06 model's age parameter works well for the age group of 27-29. This is not surprising since the average observer age in the Stiles-Burch study, on which CIEPO06 is based, was 32. For Group 3, concerning aged observers, CIEPO06 performs worse than the standard colorimetric observer for $\bar{x}_{10}(\lambda)$ and $\bar{y}_{10}(\lambda)$ CMFs. The errors in the original model prediction are comparable to the intragroup standard deviation, indicating that the prediction errors are statistically significant.

As far as the $\bar{z}_{10}(\lambda)$ CMF is concerned, the CIEPO06 model produces markedly better results compared to the CIE $10^{\circ}$ standard colorimetric observer, even without age correspondence. On an average, the reduction in mean absolute difference is more than $50 \% . \bar{z}_{10}(\lambda) \mathrm{CMF}$ also shows high standard deviation compared to $\bar{x}_{10}(\lambda)$ and $\bar{y}_{10}(\lambda)$, indicating that the high prediction error of the standard colorimetric observer is, at least partially, due to observers having short-wavelength cone sensitivity significantly different from the average. As explained in Subsection 2.D, there is high variability in ocular media optical density among observers, which is more pronounced among higher-age-group observers [39]. Presumably, this variability will manifest more significantly in the blue region of color space. It is logical to hypothesize that, in the process of averaging over the whole population of all ages, observers significantly different from the majority unduly affect the average. Within the constraints of the current analysis, CIEPO06 seems to offer an improvement over the $10^{\circ}$ standard colorimetric observer in predicting intra-age-group average $z$ functions. 

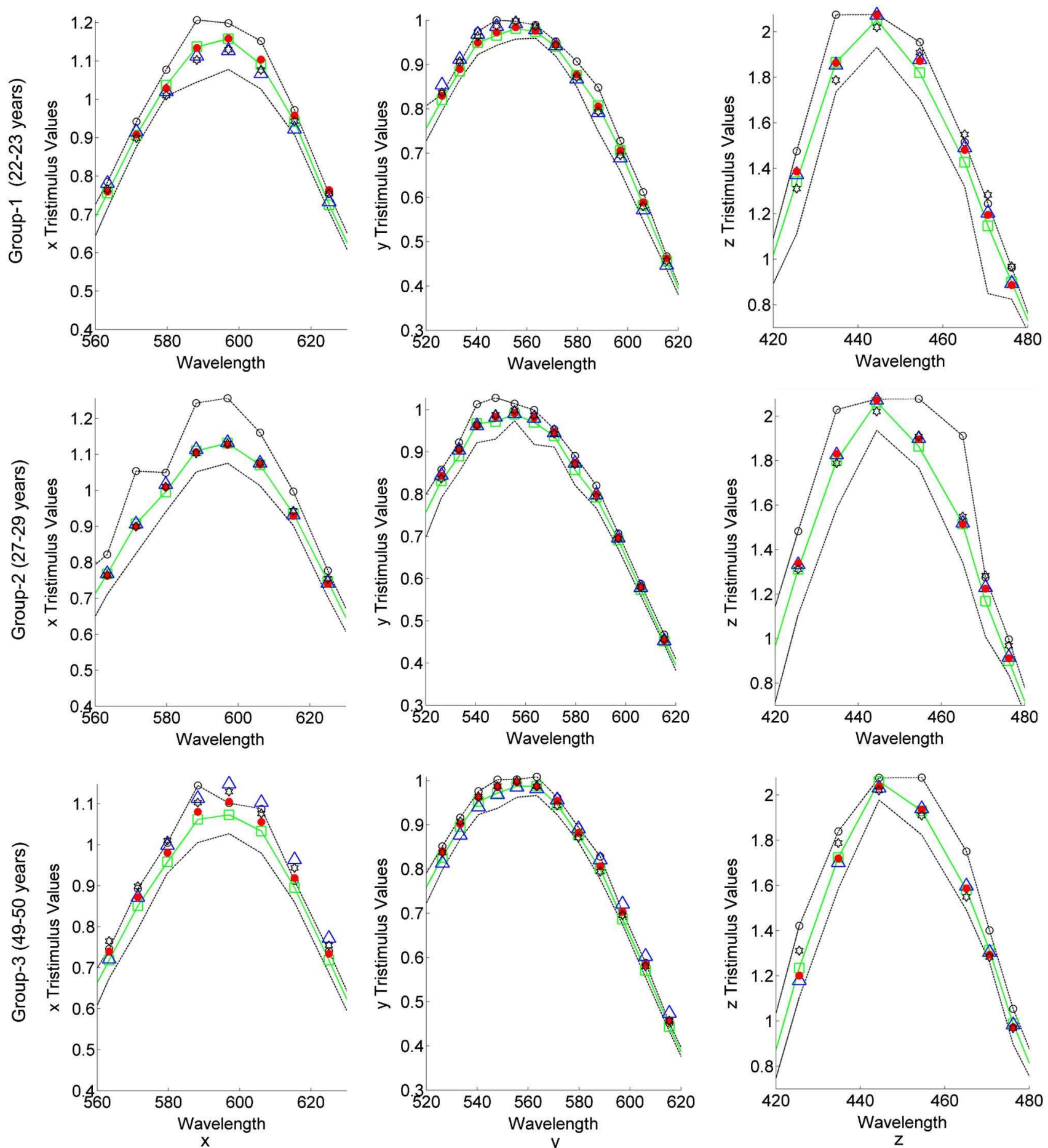

Fig. 10. (Color online) CMFs for the Stiles-Burch intragroup average observer (green curve with squares), CIEPO06 model predictions (blue triangles), CIEPO06 model predictions with age correspondence (red solid circles) and CIE $10^{\circ}$ standard colorimetric observer (black stars) for Groups 1 (top row), 2 (middle row), and 3 (bottom row). Stiles-Burch observers' intragroup minimum (solid black curve) and maximum (black curve with open circles) are also shown. Each plot shows the CMFs around the peak only.

\section{DISPLAY COLORIMETRY: COMPARISON OF CIEPO06 CMFS AND THE CIE 10 ${ }^{\circ}$ STANDARD COLORIMETRIC OBSERVER}

Any statistical method used to compare the model predictions with real observer data is incomplete without an analysis of the perceptual effect of the prediction errors. Thus, an additional analysis was performed to simulate the effect of the deviations of CIEPO06 model predictions and the CIE $10^{\circ}$ standard colorimetric observer from the average intragroup observer data on display color perception. The same method of computation of $\left(u^{\prime}, v^{\prime}\right)$ tristimulus values for the seven test stimuli was followed as was used for analyzing the effect of various psychological factors described in Subsection 2.B. The only difference in this case is in the last step. The spectral power distributions of the test stimuli, obtained from the channel values and the spectral data of the display primaries, were integrated with 
Table 3. Deviations of CMF Data from Intragroup Average Stiles-Burch Observer, $1^{\circ}$ Standard Colorimetric Observer, and CIEPO06 Model Predictions with Age Correspondence and with Real Ages

\begin{tabular}{|c|c|c|c|c|c|}
\hline \multirow[b]{3}{*}{$\mathrm{CMF}$} & \multirow[b]{3}{*}{$\begin{array}{l}\text { Grp. } \\
\text { No. }\end{array}$} & \multirow{3}{*}{$\begin{array}{c}\text { Mean } \\
\text { Intragroup } \\
\text { Stiles- } \\
\text { Burch } \\
\text { Std. Dev. }\end{array}$} & \multicolumn{3}{|c|}{$\begin{array}{l}\text { Mean Scaled Abs. Diff. } \\
\text { From Mean Intragroup } \\
\text { Stiles-Burch Data }\end{array}$} \\
\hline & & & \multirow{2}{*}{$\begin{array}{l}\text { CIE } 10^{\circ} \\
\text { Std. Col. } \\
\text { Obs. }\end{array}$} & \multicolumn{2}{|c|}{ CIEPO06 } \\
\hline & & & & $\begin{array}{c}\text { Model with } \\
\text { Real Ages }\end{array}$ & $\begin{array}{l}\text { Model with } \\
\text { Age Corres. }\end{array}$ \\
\hline \multirow[t]{3}{*}{$\bar{x}(\lambda)$} & 1 & 10.11 & 5.68 & 6.53 & 2.51 \\
\hline & 2 & 11.28 & 2.54 & 1.74 & 1.99 \\
\hline & 3 & 9.12 & 9.93 & 10.58 & 6.06 \\
\hline \multirow[t]{3}{*}{$\bar{y}(\lambda)$} & 1 & 6.02 & 2.81 & 4.73 & 1.13 \\
\hline & 2 & 6.68 & 2.28 & 2.42 & 2.43 \\
\hline & 3 & 5.41 & 2.12 & 4.21 & 2.5 \\
\hline \multirow[t]{3}{*}{$\bar{z}(\lambda)$} & 1 & 22.7 & 19.25 & 8.22 & 7.55 \\
\hline & 2 & 25.54 & 10.88 & 6.2 & 6.17 \\
\hline & 3 & 21.43 & 11.71 & 5.21 & 3.99 \\
\hline
\end{tabular}

either the CIEPO06 CMFs with age correspondence, the CIEPO06 CMFs with real ages, or the CIE $10^{\circ}$ standard colorimetric observer to obtain the $\left(u^{\prime}, v^{\prime}\right)$ specification. $\left(u^{\prime}, v^{\prime}\right)$ RMS distances were computed between coordinates corresponding to Stiles-Burch intragroup average $\left(u_{\mathrm{av}, \mathrm{SB}}^{\prime}, v_{\mathrm{av}, \mathrm{SB}}^{\prime}\right)$ and those corresponding to various model predictions ( $\left.u_{\text {pred }}^{\prime}, v_{\text {pred }}^{\prime}\right)$, as shown in Eq. (12). In this equation, the distances are normalized by $\left(u_{\text {av.SB }}^{\prime}, v_{\text {av.SB }}^{\prime}\right)$, the coordinates for Stiles-Burch intragroup average data. Such normalization allows us a comparison of relative magnitudes of various distances:

$$
\mathrm{rms}=100 \cdot \sqrt{\left(\frac{u_{\mathrm{pred}}^{\prime}-u_{\mathrm{av}, \mathrm{SB}}^{\prime}}{u_{\mathrm{av}, \mathrm{SB}}^{\prime}}\right)^{2}+\left(\frac{v_{\mathrm{pred}}^{\prime}-v_{\mathrm{av}, \mathrm{SB}}^{\prime}}{v_{\mathrm{av}, \mathrm{SB}}^{\prime}}\right)^{2}} .
$$

Table 4 lists these normalized distances computed for the LCD. For the CRT, the RMS distance differences between chromaticities predicted by the $\mathrm{CIE} 10^{\circ}$ standard colorimetric observer and the CIEPO06 model were less apparent and are not shown. Note that all these distances are computational color differences between actual and model-predicted chromaticities, and simply help us compare model prediction errors in a perceptual space. The distances in different parts of the color space are not comparable since the $\left(u^{\prime}, v^{\prime}\right)$ space is not perceptually uniform, but small distances corresponding to various CMFs can be compared. So the values in Table 4 should be compared row-wise, and not column-wise.

The shaded entries in Table 4 represent the cases where the original CIEPO06 model with real ages predicted the intragroup averages better than those of the CIE $10^{\circ}$ standard colorimetric observer (italics), as well as cases where CIEPO06 model with age correspondence predicted the intragroup averages better than the original CIEPO06 model (bold). While for Groups 1 and 3, the original CIEPO06 model predictions are generally better than the CIE $10^{\circ}$ standard colorimetric observer, the model mostly performs worse in the case of Group 2. Applying the age correspondence generally improves the model prediction in case of Groups 1 and 2. For Group 3, however, age correspondence mostly degrades the original model prediction quite significantly. This shows that reducing the overall RMS error in the cone fundamental or tristimulus space does not necessarily result in improved prediction of color perception in a chromaticity space. Another possible explanation is that the observer variability in higherage-group observers is not well modeled in CIEPO06 (see Fig. 10), thus intragroup average prediction is adversely affected by the poor prediction of color matches for observers significantly different from the average.

Now, how could we correlate the observations from Table 3 (see Subsection 3.D) and Table $\underline{4}$ ? Note that Table $\underline{3}$ lists scaled prediction errors around the peak regions of individual $x, y$, and $z$ CMFs, while Table $\underline{4}$ lists normalized RMS distances in predicting several test stimuli reproduced on the LCD in two-dimensional $\left(u^{\prime}, v^{\prime}\right)$ chromaticity space. Although it is not surprising that the observations are not always congruent with each other, two inferences can be drawn by taking into account results from both analyses.

Overall, the CIEPO06 model in its original form does not always offer an improvement over the $10^{\circ}$ standard colorimetric observer in predicting intra-age-group average observer data. Using values different from actual observer ages in the CIEPO06 model can achieve better overall correlation between actual and model-predicted CMFs in the tristimulus or cone fundamental space, but does not necessarily result in

Table 4. $\left(u^{\prime}, v^{\prime}\right)$ Normalized RMS Distances $(\times 100)$ of Predicted Chromaticity Values from Stiles-Burch Intragroup Average CMFs, Computed for Seven Test Stimuli as Viewed on the LCD ${ }^{a}$

\begin{tabular}{|c|c|c|c|c|c|c|c|c|c|}
\hline \multirow[b]{2}{*}{$\begin{array}{l}\text { Test } \\
\text { Stim. }\end{array}$} & \multicolumn{3}{|c|}{ Group 1} & \multicolumn{3}{|c|}{ Group 2} & \multicolumn{3}{|c|}{ Group 3} \\
\hline & $\begin{array}{c}\text { CIE } 10^{\circ} \\
\text { Std. Col. } \\
\text { Obs. }\end{array}$ & $\begin{array}{c}\text { CIEPO06 } \\
\text { with real } \\
\text { ages }\end{array}$ & $\begin{array}{c}\text { CIEPO06 } \\
\text { With Age } \\
\text { Corres. }\end{array}$ & $\begin{array}{c}\text { CIE } 10^{\circ} \\
\text { Std. Col. } \\
\text { Obs. }\end{array}$ & $\begin{array}{c}\text { CIEPO06 } \\
\text { with real } \\
\text { ages }\end{array}$ & $\begin{array}{c}\text { CIEPO06 } \\
\text { with Age } \\
\text { Corres. }\end{array}$ & $\begin{array}{c}\text { CIE } 10^{\circ} \\
\text { Std. Col. } \\
\text { Obs. }\end{array}$ & $\begin{array}{c}\text { CIEPO06 } \\
\text { with real } \\
\text { ages }\end{array}$ & $\begin{array}{c}\text { CIEPO06 } \\
\text { with Age } \\
\text { Corres. }\end{array}$ \\
\hline TS-1 & 4.52 & 5.23 & 2.00 & 2.89 & 2.80 & 2.43 & 1.79 & 0.41 & 3.70 \\
\hline TS-2 & 2.36 & 1.85 & 1.90 & 1.47 & 1.84 & 1.74 & 1.77 & 1.79 & 1.83 \\
\hline TS-3 & 1.11 & 0.49 & 1.40 & 0.82 & 1.16 & 1.22 & 1.46 & 1.89 & 0.79 \\
\hline $\mathrm{TS}-4$ & 4.19 & 4.34 & 0.61 & 2.68 & 2.72 & 1.84 & 0.81 & 0.63 & 4.62 \\
\hline TS-5 & 1.97 & 0.81 & 0.92 & 1.29 & 1.91 & 1.42 & 1.30 & 2.15 & 2.59 \\
\hline TS-6 & 3.54 & 3.13 & 1.15 & 2.27 & 2.64 & 1.23 & 1.80 & 1.47 & 5.42 \\
\hline TS-7 & 3.51 & 3.35 & 1.48 & 2.22 & 2.43 & 1.96 & 1.37 & 0.68 & 3.35 \\
\hline
\end{tabular}

${ }^{a}$ Predicted chromaticity values were obtained using CIE $10^{\circ}$ standard colorimetric observer CMFs, CIEPO06 model CMFs with real ages, and CIEPO06 model CMFs with age correspondence. Highlighted values indicate improvement in the prediction of chromaticities corresponding to intragroup average CMFs, either by the CIEPO06 original model compared to the CIE $10^{\circ}$ standard colorimetric observer (italics), or by the CIEPO06 model with age correspondence compared to the original CIEPO06 model (bold). 
improved prediction of individual color matches, particularly when the stimuli do not have flat spectral characteristics. While the short-wavelength CIEPO06 CMFs consistently perform better than the $10^{\circ}$ standard colorimetric observer for all three age groups, the model's prediction errors in medium and long wavelengths are significantly higher for Group 3. Why does the model not work well for higher-age-group StilesBurch observers at longer wavelengths? This issue is further investigated in the next section.

\section{OPTIMIZED CIEPO06 CONE FUNDAMENTALS FOR STILES-BURCH OBSERVER GROUPS}

As discussed in the preceding sections, the CIEPO06 model does not satisfactorily predict the intragroup Stiles-Burch average observer CMFs $\bar{x}_{10}(\lambda)$ and $\bar{y}_{10}(\lambda)$ in the long- and medium- wavelength ranges, particularly for higher-age-group observers. These observations thus raise the question: can we improve the model performance in the longer wavelengths? If so, how can we achieve that?

This prediction error can result from many potential sources. For example, it could be due to individual observer's LWS or MWS photopigment peak-wavelength shift resulting from genetic polymorphism (as discussed in Section 2), or it could be due to poor modeling of cone absorptance spectra in longer wavelengths. As far as the prediction error at higher wavelengths is concerned, we can rule out the role of ocular media and macular pigment optical density factors, since their influences are insignificant beyond $550 \mathrm{~nm}$. Note that ocular media optical density is the only physiological factor in the CIEPO06 model that changes with age. To probe possible ways to improve the CIEPO06 model prediction at higher wavelengths, a constrained nonlinear optimization was performed under two different conditions.

\section{A. Method of Optimization}

In the first case, only the peak wavelength shifts of the LWS or MWS photopigments were allowed to vary, keeping all other parameters constant. In the second case, a weighting function for the low-optical-density absorption spectra was introduced, which was then optimized. In both cases, the original CIEPO06 functions at the short wavelengths were not altered.

The equations for CIEPO06 cone fundamentals were introduced in Eq. (3). In the first optimization, only the peak wavelength shifts of $A_{l}(\lambda)$ and $A_{m}(\lambda)$ functions were allowed to vary, keeping all other parameters constant. The optimized cone fundamentals can thus be represented by Eq. (13):

$$
\begin{aligned}
\bar{l}_{\text {opt }}(\lambda) & =\left[1-10^{-D_{\text {vis }, l} \cdot A_{\text {shifted }, l}(\lambda)}\right] \cdot 10^{-D_{\text {mac }}(\lambda)} \cdot 10^{-D_{\text {ocul }}(\lambda)}, \\
\bar{m}_{\text {opt }}(\lambda) & =\left[1-10^{-D_{\text {vis }, m} \cdot A_{\text {shifted }, m}(\lambda)}\right] \cdot 10^{-D_{\text {mac }}(\lambda)} \cdot 10^{-D_{\text {ocul }}(\lambda)} .
\end{aligned}
$$

Here, the $\left[D_{\text {vis }, l} A_{\text {shifted }, l}(\lambda)\right]$ and $\left[D_{\text {vis }, m} A_{\text {shifted, } m}(\lambda)\right]$ terms are mathematical representations of the peak wavelength shift due to polymorphism. In the actual implementation of the optimization method, the peak wavelength $\lambda$ was first shifted in the wavenumber scale $\left(\nu=10^{7} / \lambda\right.$, where $\nu$ is in $\mathrm{cm}^{-1}$ and $\lambda$ is in nanometers) independently for LWS and MWS photopigment. Next, the cone absorptance spectra were resampled, then modified cone fundamentals were computed and converted from the quanta to energy units, and finally were renormalized. In the objective function, the RMSE over the whole wavelength range was computed between the modified CIEPO06 cone fundamentals and Stiles-Burch intragroup average cone fundamentals, and was minimized iteratively by changing the amount of peak $\lambda$ shift. This shift was constrained between +250 and $-250 \mathrm{~cm}^{-1}$, with a starting value of $100 \mathrm{~cm}^{-1}$. Thus, the optimization process left the contributions of macular pigment and ocular media unaltered; only the contributions of LWS and MWS cone absorption spectra were changed. The SWS cone fundamental was not modified. The optimization was terminated after 10,000 iterations, or below an error of $10^{-6}$, whichever was earlier.

In the second optimization, weighting functions $w_{l}(\lambda)$ and $w_{m}(\lambda)$ for the low-optical-density spectral absorbance terms $A_{l}(\lambda)$ and $A_{m}(\lambda)$, respectively, were introduced beyond $550 \mathrm{~nm}$ [Eq. (14)]. As before, the SWS cone fundamental was unaltered:

$$
\begin{aligned}
\bar{l}_{\mathrm{opt}}(\lambda) & =\left[1-10^{-D_{\mathrm{vis}, l} \cdot A_{l}(\lambda) \cdot w\left(\lambda_{l}\right)}\right] \cdot 10^{-D_{\mathrm{mac}}(\lambda)} \cdot 10^{-D_{\mathrm{ocul}}(\lambda)}, \\
\bar{m}_{\mathrm{opt}}(\lambda) & =\left[1-10^{-D_{\mathrm{vis}, m} \cdot A_{m}(\lambda) \cdot w\left(\lambda_{m}\right)}\right] \cdot 10^{-D_{\text {mac }}(\lambda)} \cdot 10^{-D_{\text {ocul }}(\lambda)} .
\end{aligned}
$$

While some authors have already questioned the CIEPO06 SWS cone fundamental at short wavelengths [40], for our work, there are two reasons for restricting optimization above $550 \mathrm{~nm}$. First, we are primarily interested in reducing prediction errors at higher wavelengths. Second, the ocular media and macular pigment optical densities have significant contributions to the cone fundamentals below $550 \mathrm{~nm}$. Thus, even if we introduce a weighting function below $550 \mathrm{~nm}$ and obtain better results, it is difficult to isolate a single physiological factor as the source of prediction error.

As in the first optimization, the RMSE between the modified CIEPO06 cone fundamentals and the Stiles-Burch intragroup average cone fundamentals were minimized in the objective function.

\section{B. Results}

As a result of the first optimization, the peak wavelengths of $A_{l}(\lambda)$ and $A_{m}(\lambda)$ functions were shifted differently for different groups. For Group 1, LWS and MWS peak wavelength shifts were 3.6 and $1.3 \mathrm{~nm}$, respectively, both toward shorter wavelengths. For Group 2, only the LWS function was shifted by $0.1 \mathrm{~nm}$ toward shorter wavelengths. For Group 3, on the other hand, the shifts were toward longer wavelengths, 4.1 and $0.3 \mathrm{~nm}$ for, respectively, LWS and MWS, functions.

The second optimization resulted in different LWS and MWS weighting functions for the three groups. These functions are shown in Fig. 11. The optimized function is obtained by multiplying the original CIEPO06 model function by the respective weighting function. Thus a weighting of unity does not affect the original model function. As shown in Fig. 11, the LWS weighting functions have higher values than those of the MWS cones. What is interesting is that, for both LWS and MWS, the weighting functions for Groups 1 and 3 are somewhat symmetrical around the unity weights. To remind the reader, these two groups consist of younger (22-23 years) and older (49-50 years) observers, respectively, while Group 2 observers have average age in the middle (27-29 years). For higher-agegroup observers, peak optical density is reduced by the optimization process, and is increased for the lower age group.

Results of both optimization processes are incorporated in Table 5, introduced earlier in Subsection 3.D (see Table 3). 

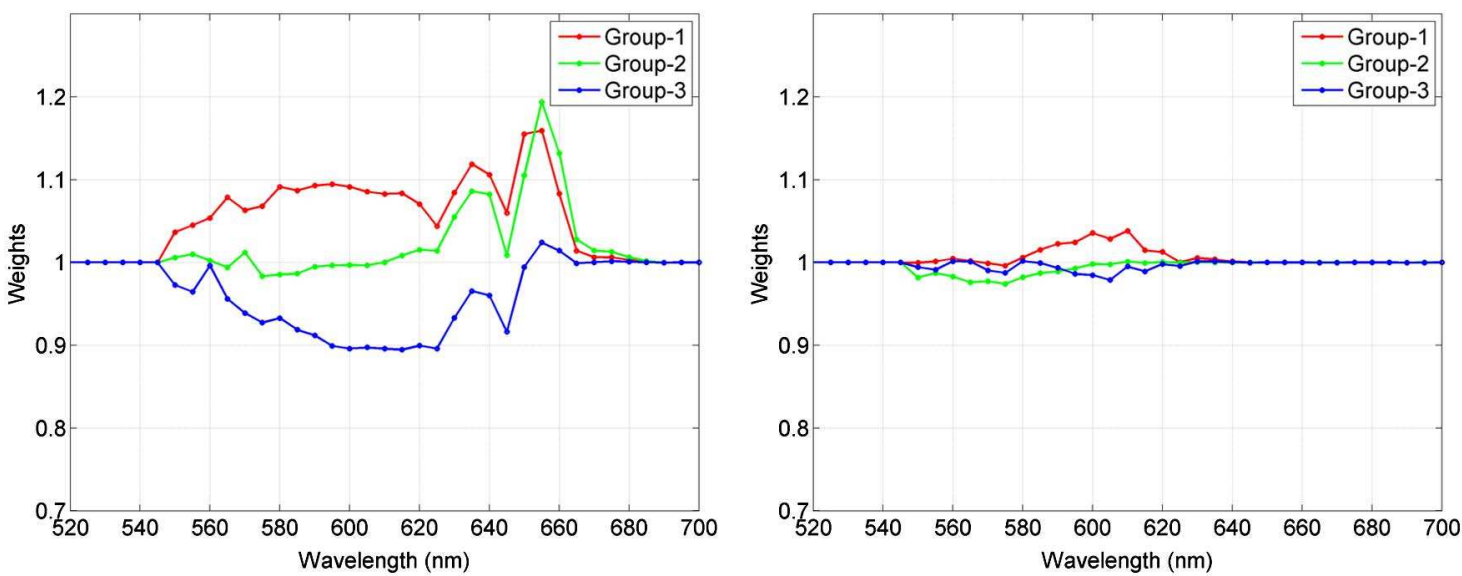

Fig. 11. (Color online) Weighting functions for optimizing the LWS (left) and MWS (right) low-density spectral absorbance. Optimization was performed above $550 \mathrm{~nm}$.

Both $\bar{x}(\lambda)$ and $\bar{y}(\lambda)$ intra-age-group average CMFs of StilesBurch observers of Groups 1 and 3 are better predicted by the optimized model.

The improvement in model performance is also substantiated in Table 6 . The shaded entries in Table $\underline{6}$ represent the cases where the original CIEPO06 model with real ages predicted the intragroup average data better than those of the CIE $10^{\circ}$ standard colorimetric observer (italics), as well as cases where the optimized CIEPO06 model predicted the intragroup averages better than the original CIEPO06 model, as well as the CIE $10^{\circ}$ standard colorimetric observer (bold). These values were computed in the same way as described in Section 4 . Overall, the peak wavelength shift optimization did not lead to better prediction of average data. An effect of polymorphism on the average data is not apparent in any of the three groups. This supports Webster's conclusion [29] that no polymorphism effect among the Stiles-Burch observers could be confirmed. However, this depends on the observer group involved in the study. Viénot et al. [25] showed that a shift in the wavelength of peak sensitivity of the cone photopigments could account for the variability in multiple Rayleigh matches from color-normal observers.

Table 5. Comparison of Deviations of CMF Data from Intragroup Average Stiles-Burch Observer, $10^{\circ}$ Standard Colorimetric Observer, CIEPO06 Original Model Predictions, and Optimized CIEPO06 Model with Modified Low-Density Absorbance Spectra

\begin{tabular}{|c|c|c|c|c|c|}
\hline \multirow[b]{3}{*}{$\mathrm{CMF}$} & \multirow[b]{3}{*}{$\begin{array}{c}\text { Group } \\
\text { No. }\end{array}$} & \multirow[b]{3}{*}{$\begin{array}{c}\text { Mean } \\
\text { Intragroup } \\
\text { Stiles- } \\
\text { Burch } \\
\text { Std. Dev. }\end{array}$} & \multicolumn{3}{|c|}{$\begin{array}{c}\text { Mean Scaled } \\
\text { Abs. Diff. From Mean } \\
\text { Intragroup Stiles-Burch Data }\end{array}$} \\
\hline & & & & \multicolumn{2}{|c|}{ CIEPO06 Model } \\
\hline & & & $\begin{array}{l}\text { CIE } 10^{\circ} \\
\text { Std. Obs. }\end{array}$ & Original & $\begin{array}{c}\text { Optimized } \\
\text { (Low Density } \\
\text { Abs. } \\
\text { Spectra) }\end{array}$ \\
\hline \multirow[t]{3}{*}{$\bar{x}(\lambda)$} & 1 & 10.11 & 5.68 & 6.53 & 2.01 \\
\hline & 2 & 11.28 & 2.54 & 1.74 & 2.17 \\
\hline & 3 & 9.12 & 9.93 & 10.58 & 2.01 \\
\hline \multirow[t]{3}{*}{$\bar{y}(\lambda)$} & 1 & 6.02 & 2.81 & 4.73 & 1.34 \\
\hline & 2 & 6.68 & 2.28 & 2.42 & 1.4 \\
\hline & 3 & 5.41 & 2.12 & 4.21 & 1.12 \\
\hline
\end{tabular}

In our analysis, significant improvement was achieved by optimizing the low-density photopigment spectral absorbance functions for Groups 1 and 3. On an average, for Group 1, the average RMS prediction error for the seven stimuli reduced by more than $70 \%$ as compared to the CIE $10^{\circ}$ standard colorimetric observer, while for Group 3, the improvement was around $45 \%$. The only exception is the blue test color (TS-6) for Group 3, which, in any case, does not have significant spectral power in the wavelengths beyond $550 \mathrm{~nm}$. In general, in the case of Group 2, the optimization did not improve the results.

\section{Discussion}

The foregoing discussion leads to a hypothesis that a major source of the CIEPO06 model prediction errors at higher wavelengths is in the model's cone absorptance spectra, which has two components, photopigment low-density spectral absorbance function and the peak optical density of visual pigment. Figure 11 indicates that cone absorptance spectra should have an age-dependent component, which would cause the cone absorptance spectra to reduce as the age is increased. This component should have different values in the long- and medium-wavelength range.

What could be the physiological explanation for such a component, which is missing from the model? As explained in Section 5.8 of the CIE TC 1-36 report [11], there are some indications that the peak optical density of the visual pigment decreases gradually as a function of age. However, because of insufficient or contradictory data to support this hypothesis [41-43], such dependence has been ignored in the model. A logical argument would be that the age dependence of this factor has a significant effect on cone fundamentals and color matches, and that its exclusion from the CIEPO06 model leads to prediction errors of intra-age-group average at higher wavelengths. This argument appears to contradict Webster and MacLeod's [44] observation that none of the factors extracted through a factor analysis of the Stiles-Burch $10^{\circ}$ data corresponded to differences in photopigment density, and only a weak role of density differences was suggested by the fits to the correlation matrix. They concluded the peak wavelength shift of photopigment density was a more salient determinant of individual differences in the matches. A key difference between that study and our analysis is that Webster and MacLeod were investigating individual variability without 
Table 6. $\left(u^{\prime}, v^{\prime}\right)$ Normalized RMS Distances (x100) from Stiles-Burch Intragroup Average Chromaticities Computed for Seven Test Stimuli as Viewed on the LCD $^{a}$

\begin{tabular}{|c|c|c|c|c|c|c|c|c|c|c|c|c|}
\hline \multirow[b]{2}{*}{$\begin{array}{l}\text { Test } \\
\text { Stim. }\end{array}$} & \multicolumn{4}{|c|}{ Group 1} & \multicolumn{4}{|c|}{ Group 2} & \multicolumn{4}{|c|}{ Group 3} \\
\hline & $\begin{array}{c}\text { CIE } 10^{\circ} \\
\text { Std. Col. } \\
\text { Obs. }\end{array}$ & CIEPO06 & $\begin{array}{c}\text { CIEPO06 } \\
\text { Optim. } \\
\text { (Peak } \lambda \\
\text { Shift) }\end{array}$ & $\begin{array}{c}\text { CIEPO06 } \\
\text { Optim. } \\
\text { (LD Abs } \\
\text { Spectra) }\end{array}$ & $\begin{array}{c}\text { CIE } 10^{\circ} \\
\text { Std. Col. } \\
\text { Obs. }\end{array}$ & CIEPO06 & $\begin{array}{l}\text { CIEPO06 } \\
\text { Optim. } \\
\text { (Peak } \lambda \\
\text { Shift) }\end{array}$ & $\begin{array}{c}\text { CIEPO06 } \\
\text { Optim. } \\
\text { (LD Abs } \\
\text { Spectra) }\end{array}$ & $\begin{array}{c}\text { CIE } 10^{\circ} \\
\text { Std. Col. } \\
\text { Obs. }\end{array}$ & CIEPO06 & $\begin{array}{l}\text { CIEPO06 } \\
\text { Optim. } \\
\text { (Peak } \lambda \\
\text { Shift) }\end{array}$ & $\begin{array}{c}\text { CIEPO06 } \\
\text { Optim. } \\
\text { (LD Abs } \\
\text { Spectra) }\end{array}$ \\
\hline TS-1 & 4.52 & 5.23 & 5.21 & 0.58 & 2.89 & 2.80 & 3.54 & 2.96 & 1.79 & 0.41 & 2.18 & 0.11 \\
\hline TS-2 & 2.36 & 1.85 & 3.88 & 0.25 & 1.47 & 1.84 & 2.23 & 1.12 & 1.77 & 1.79 & 2.23 & 0.26 \\
\hline TS-3 & 1.11 & 0.49 & 2.86 & 0.72 & 0.82 & 1.16 & 1.08 & 0.11 & 1.46 & 1.89 & 1.60 & 0.46 \\
\hline TS-4 & 4.19 & 4.34 & 3.99 & 0.86 & 2.68 & 2.72 & 3.06 & 2.84 & 0.81 & 0.63 & 0.97 & 0.73 \\
\hline TS-5 & 1.97 & 0.81 & 2.70 & 0.77 & 1.29 & 1.91 & 2.25 & 1.30 & 1.30 & 2.15 & 1.78 & 1.10 \\
\hline TS-6 & 3.54 & 3.13 & 2.47 & 0.86 & 2.27 & 2.64 & 2.36 & 2.43 & 1.80 & 1.47 & 2.16 & 2.36 \\
\hline TS-7 & 3.51 & 3.35 & 4.18 & 0.52 & 2.22 & 2.43 & 2.95 & 2.23 & 1.37 & 0.68 & 1.76 & 0.36 \\
\hline
\end{tabular}

${ }^{a}$ Results are shown for the CIE $10^{\circ}$ standard colorimetric observer, original CIEPO06 model predictions and optimized model prediction through peak-wavelength shift and weighted low-density photopigment spectral absorbance. Highlighted values indicate improvement in the prediction of chromaticities corresponding to intragroup average CMFs, either by the CIEPO06 original model compared to the CIE $10^{\circ}$ standard colorimetric observer (italics), or by the optimized CIEPO06 model compared to both original CIEPO06 model and CIE $10^{\circ}$ standard colorimetric observer (bold).

regard to age groups, while we focused on intra-age-group average prediction. For the latter, differences in photopigment optical density does seem to be an important factor.

It should be emphasized that the optimization method described in this section is purely mathematical. Deriving a physiologically based correction function was beyond the scope of the current study. However, we believe this analysis isolates the likely source of a major flaw in the CIEPO06 model, correcting which can lead to a significant improvement in model performance, particularly for observers in higher age groups compared to the Stiles-Burch observers' average age of 32 .

\section{CONCLUSIONS}

In this paper, we presented a theoretical analysis on various aspects of the physiologically based observer model proposed by CIE TC 1-36 (CIEPO06). In the context of color perception on modern narrowband displays, we evaluated the performance of the CIEPO06 model in predicting the average data for three different age groups of Stiles-Burch observers and compared the results with the CIE $10^{\circ}$ standard colorimetric observer. Our goal was to determine if an age-dependent observer provides an advantage over a single average observer. Several conclusions can be drawn from our study as listed below.

i. The photopigment peak optical density has the strongest influence in display color perception compared to other physiological factors. This finding assumes further significance in light of Smith et al.'s [45] observation that a variation of \pm 0.2 unit of photopigment optical density from the mean could account for $99 \%$ of the individual variance in the Stiles-Burch pilot data [9]. Photopigment peak wavelength shift is another factor having significant contribution to observer variability, but is not within the scope of the CIEPO06 model.

ii. Using real observer ages in the model leads to large errors in intra-age group average observer CMF prediction, making it difficult to apply this model directly in practical applications.

iii. The CIE $10^{\circ}$ standard colorimetric observer $z$ function has a large error with respect to intra-age-group average $z$ functions of all three Stiles-Burch age groups studied, namely six, 10, and six observers in age ranges or 22-23, 27-29, and 49-50 years, respectively; in all three cases, the CIEPO06 model provides significant improvement.

iv. $x$ and $y$ CMFs derived from the CIEPO06 model for the observer age group of 49-50 years show high deviation from the intragroup average, the error being comparable to intragroup standard deviation.

v. In terms of predicting average color perception for different age groups on a display with narrowband primaries, the CIEPO06 model in its original form does not always offer an improvement over the $10^{\circ}$ standard colorimetric observer. This limitation is particularly apparent for higher age group observers in the red-green part of the color space.

vi. A constrained nonlinear optimization of the CIEPO06 model shows that only peak wavelength shifts of the LWS and MWS photopigment density fails to improve intra-age-group average prediction, while weighting functions for the photopigment density functions above $550 \mathrm{~nm}$ significantly improve this prediction both in the spectral domain and chromaticity space, for both age groups of 22-23 and 49-50 years. This weighting function is different for different age groups and also different for LWS and MWS cone photopigment densities. We propose that the peak optical density of visual pigments should be an age-dependent function in the CIEPO06 model and should be defined independently for LWS and MWS cone photopigments.

\section{REFERENCE}

1. W. D. Wright, "A re-determination of the trichromatic coefficients of the spectral colors," Trans. Opt. Soc. 30, 141-164 (1929).

2. J. Guild, "The colorimetric properties of the spectrum," Phil. Trans. R. Soc. A 230, 149-187 (1932).

3. CIE, Sixième Session, Genève, Juillet, 1924, Recueil des Travaux et Compte Rendu de Séances (Cambridge University, 1926), pp 67-69.

4. R. S. Berns, Billmeyer and Saltzman's Principles of Color Technology, 3rd ed. (Wiley, 2000).

5. V. C. Smith and J. Pokorny, "Color matching and color discrimination," in The Science of Color, 2nd ed, S. K. Shevell, ed. (Elsevier, , 2003), pp. 103-148.

6. D. B. Judd, "Colorimetry and artificial daylight," in Technical Committee No. 7 Report of Secretariat United States Commission (International Commission on Illumination, 1951), pp. 1-60. 
7. J. J. Vos, "Colorimetric and photometric properties of a $2^{\circ}$ fundamental observer," Color Res. Appl. 3, 125-128 (1978).

8. CIE 1988, " $2{ }^{\circ}$ spectral luminous efficiency function for photopic vision,” CIE 86-1990 (Commission Internationale de l'Éclairage, 1990).

9. W. S. Stiles and J. M. Burch, "N.P.L. colour-matching investigation: final report," Opt. Acta 6, 1-26 (1959).

10. N. I. Speranskaya, "Determination of spectral color co-ordinates for twenty-seven normal observers," Opt. Spectrosc. 7, 424-428 (1959).

11. "Fundamental chromaticity diagram with physiological axesPart I," CIE Technical Report 170-1 (CIE, 2006).

12. A. Stockman and L. T. Sharpe, "The spectral sensitivities of the middle- and long- wavelengthsensitive cones derived from measurements in observers of known genotype," Vision Res. 40, 1711-1737 (2000).

13. A. Stockman, D. I. A. MacLeod, and N. E. Johnson, "Spectral sensitivities of human cones," J. Opt. Soc. Am. A 10, 2491-2521 (1993).

14. D. I. A. MacLeod and M. A. Webster, "Factors influencing the color matches of normal observers," in Colour Vision: Physiology and Psychophysics, J. D. Mollon and L. T. Sharpe, eds. (Academic, 1983), pp. 81-92.

15. V. C. Smith, J. Pokorny, and Q. Zaidi, "How do sets of colormatching functions differ?" in Colour Vision: Physiology and Psychophysics, J. D. Mollon and L. T. Sharpe, eds. (Academic, 1983).

16. F. Viénot, "Relations between inter- and intra-individual variability of color matching functions. experimental results," J. Opt. Soc. Am. 70, 1476-1483 (1980).

17. G. Wyszecki and W. S. Stiles, Color Science: Concepts and Methods, Quantitative Data and Formulae, 2nd ed. (Wiley, 1982).

18. A. North and M. Fairchild, "Measuring color-matching functions. Part I," Color Res. Appl. 18, 155-162 (1993).

19. A. North and M. Fairchild, "Measuring color-matching functions. Part II. New data for assessing observer metamerism," Color Res. Appl. 18, 163-170 (1993).

20. B. Oicherman, M. R. Luo, B. Rigg, and A. R. Robertson, "Effect of observer metamerism on colour matching of display and surface colours," Color Res. Appl. 33, 346-359 (2008).

21. C. Oleari and M. Pavesi, "Grassmann's laws and individual color-matching functions for nonspectral primaries evaluated by maximum saturation technique in foveal vision," Color Res. Appl. 33, 271-281 (2008).

22. Y. Nakano, Y. Nakayasu, H. Morita, K. Suehara, J. Kohda, and T. Yano, "Individual difference of color matching functions and its cause," presented at the ISCC/CIE Expert Symposium, Ottawa, Ontario, Canada, 16-17 May 2006.

23. J. Neitz and G. H. Jacobs, "Polymorphism in normal human color vision and its mechanism," Vision Res. 30, 621-636 (1990).

24. J. C. He and S. K. Shevell, "Individual differences in cone photopigments of normal trichromats measured by dual Raleigh-type color matches," Vision Res. 34, 367-376 (1994).

25. F. Viénot, L. Serreault, and P. P. Fernandez, "Convergence of experimental multiple Rayleigh matches to peak L- and Mphotopigment sensitivity estimates," Visual Neurosci. 23, 1-8 (2006).

26. J. L. Barbur, M. Rodriguez-Carmona, J. A. Harlow, K Mancuso, J. Neitz, and M. Neitz, "A study of unusual Rayleigh matches in deutan deficiency," Visual Neurosci. 25, 507-516 (2008).

27. P. B. M. Thomas and J. D. Mollon, "Modelling the Rayleigh match," Visual Neurosci. 21, 477-482 (2004).

28. P. Csuti and J. Schanda, "Colour matching experiments with RGB-LEDs," Color Res. Appl. 33, 108-112 (2008).

29. M. A. Webster, "Reanalysis of $\lambda_{\max }$ variations in the Stiles-Burch $10^{\circ}$ color-matching functions," J. Opt. Soc. Am. A 9, 1419-1421 (1992).

30. L. T. Sharpe, A. Stockman, H. Jägle, and J. Nathans, "Opsin genes, cone photopigments, color vision, and color blindness," in Color Vision: From Genes to Perception, 1st ed., K. R. Gegenfurtner, L. T. Sharpe, and B. B. Boycott, eds. (Cambridge University, 2001), pp. 3-52.

31. A. Sarkar, L. Blondé, P. L. Callet, F. Autrusseau, J. Stauder, and P. Morvan, "Study of observer variability in modern display colorimetry: an analysis of CIE 2006 model," in Proceedings of the 11th Congress of the International Colour Association (AIC), D. Smith, P. Green-Armytage, M. A. Pope, and N. Harkness, eds. (CD) (Colour Society of Australia, 2009).

32. V. C. Smith and J. Pokorny, "Chromatic-discrimination axes, CRT phosphor spectra and individual variation in color vision," J. Opt. Soc. Am. A 12, 27-35 (1995).

33. O. Packer and D. R. Williams, "Light, the retinal image and photoreceptors," in The Science of Color, 2nd ed., S. K. Shevell, ed. (Elsevier, 2003), pp. 41-102.

34. V. C. Smith and J. Pokorny, "The design and use of a conechromaticity space," Color Res. Appl. 21, 375-383 (1996).

35. E. Miyahara, V. C. Smith, and J. Pokorny, "How surrounds affect chromatic discrimination," J. Opt. Soc. Am. A 10, 545-553 (1993).

36. D. I. A. MacLeod and R. M. Boynton, "Chromaticity diagram showing cone excitation by stimuli of equal luminance," J. Opt. Soc. Am. A 69, 1183-1185 (1979).

37. R. M. Boynton and N. Kambe, "Chromatic difference steps of moderate size measured along theoretically critical axes," Color Res. Appl. 5, 13-23 (1980).

38. A. Stockman, Colour \& Vision Research Laboratory website, http://www.cvrl.org/.

39. J. Pokorny, V. C. Smith, and M. Lutze, "Aging of the human lens," Appl. Opt. 26, 1437-1440 (1987).

40. P. Csuti and J. Schanda, "A better description of metameric experience of LED clusters," in Proceedings of Light and Lighting Conference with Special Emphasis on LEDs and Solid State Lighting (Commission Internationale de l'Éclairage, 2009).

41. A. E. Elsner, L. Berk, S. A. Burns, and P. R. Rosenberg, "Aging and human cone photopigments," J. Opt. Soc. Am. A 5, 2106-2112 (1988).

42. W. H. Swanson and G. E. Fish, "Age-related changes in the colour-match-area effect," Vision Res. 36, 2079-2085 (1996).

43. A. B. Renner, H. Knau, M. Neitz, J. Neitz, and J. S. Werner "Photopigment optical density of the human foveola and a paradoxical senescent increase outside the fovea," Visual Neurosci, 21, 827-834 (2004).

44. M. A. Webster and D. I. A. MacLeod, "Factors underlying individual-differences in the color matches of normal observers," J. Opt. Soc. Am. A 5, 1722-1735 (1988).

45. V. C. Smith, J. Pokorny, and S. J. Starr, "Variability of color mixture data-I. Interobserver variability in the unit coordinates," Vision Res. 16, 1087-1094 (1976). 